\title{
Supersymmetric $t-J$ models with long-range interactions: partition function and spectrum
}

\author{
B. Basu-Mallick ${ }^{1}$, N. Bondyopadhaya ${ }^{2}$, J.A. Carrasco ${ }^{3}$, \\ F. Finkel ${ }^{3}$, A. González-López ${ }^{3}$ \\ ${ }^{1}$ Theory Division, Saha Institute of Nuclear Physics, HBNI, 1/AF Bidhan Nagar, \\ Kolkata 700 064, India \\ ${ }^{2}$ Integrated Science Education and Research Centre, Siksha-Bhavana, Visva-Bharati, \\ Santiniketan 731 235, India \\ ${ }^{3}$ Departamento de Física Teórica, Universidad Complutense de Madrid, 28040 \\ Madrid, Spain \\ E-mail: bireswar.basumallick@saha.ac.in, \\ nilanjan.iserc@visva-bharati.ac.in, joseacar@ucm.es, ffinkel@ucm.es, \\ artemio@ucm.es
}

\begin{abstract}
.
We study the spectrum of the long-range supersymmetric $\operatorname{su}(m) t-J$ model of Kuramoto and Yokoyama in the presence of an external magnetic field and a charge chemical potential. To this end, we first establish the precise equivalence of a large class of models of this type to a family of $\mathrm{su}(1 \mid \mathrm{m})$ spin chains with long-range exchange interactions and a suitable chemical potential term. We exploit this equivalence to compute in closed form the partition function of the long-range $t$ - $J$ model, which we then relate to that of an inhomogeneous vertex model with simple interactions. From the structure of this partition function we are able to deduce an exact formula for the restricted partition function of the long-range $t$ - $J$ model in subspaces with well-defined magnon content in terms of its analogue for the equivalent vertex model. This yields a complete analytical description of the spectrum in the latter subspaces, including the precise degeneracy of each level, by means of the supersymmetric version of Haldane's motifs and their related skew Young tableaux. As an application, we determine the structure of the motifs associated with the ground state of the spin $1 / 2$ model in the thermodynamic limit in terms of the magnetic field strength and the charge chemical potential. This leads to a complete characterization of the distinct ground state phases, determined by their spin content, in terms of the magnetic field strength and the charge chemical potential.
\end{abstract}

Keywords: integrable spin chains and vertex models, solvable lattice models 


\section{Introduction}

Among lattice models of strongly correlated fermions, the $t$ - $J$ model holds a prominent position due to its role in the theoretical description of high-temperature superconductivity and as an example of a simple model featuring spin-charge separation [1-3]. In this model each lattice site can be either empty or occupied by one fermion, which interacts with its nearest neighbors through spin exchange and charge repulsion and can also hop between contiguous lattice sites.. In the one-dimensional case the $t-J$ model is of particular interest, as it is both supersymmetric and exactly solvable through the nested Bethe ansatz when its parameters are suitably related $[1,4-8]$. In the early nineties, Kuramoto and Yokoyama [9, 10] introduced a long-range version of the supersymmetric $t-J$ model featuring $1 / r^{2}$ interactions, which reduces to the $\mathrm{su}(2)$ Haldane-Shastry (HS) chain $[11,12]$ in the high density limit (i.e., when all the sites are occupied). Among other interesting features, the Kuramoto-Yokoyama (KY) model exhibits strong spin-charge separation, in the sense that at low temperatures the spin and charge velocities are respectively independent of the charge density and the magnetization. At low energies the KY model is known to be a Luttinger liquid [13], with spin and charge excitations independently described by a $c=1$ conformal field theory (CFT).

The supersymmetric KY model is exactly solvable through the asymptotic Bethe ansatz pioneered by Sutherland and Shastry [14], as its energies can be found in principle by solving a system of transcendental equations in the asymptotic momenta $[15,16]$. However, this method does not completely determine the spectrum, since it does not provide complete information on the degeneracy of each level. Based on numerical calculations, Wang et al. [17] proposed an empirical description of the degeneracies of the spectrum of the $\mathrm{su}(2) \mathrm{KY}$ model reminiscent of the rule for filling the border strips associated to Haldane's motifs [18-20]. This description, however, is known to be incorrect in certain situations, although the needed corrections vanish in the thermodynamic limit [17]. Inspired by the equivalence between the $\mathrm{su}(2) \mathrm{KY}$ model and the $\mathrm{su}(1 \mid 2)$ supersymmetric HS chain (up to a term proportional to the total electric charge), Saiga and Kuramoto [21] conjectured a description of the former model's spectrum essentially in terms of $\mathrm{su}(1 \mid 2)$-supersymmetric Haldane motifs, which accounted for the numerical results for $N \leqslant 16$ spins. To the best of our knowledge, this conjecture has remained unproved in the literature.

In this paper we address and completely solve the problem of finding a full description of the spectrum of the supersymmetric $\mathrm{su}(m) \mathrm{KY}$ model with a general chemical potential term for a finite number of sites, including the determination of the levels' degeneracies and spin content. In particular, our results provide a rigorous proof of the Saiga-Kuramoto conjecture for arbitrary $m$ and $N$. Our approach, which we shall now briefly summarize, is new and bypasses the usual machinery of the asymptotic Bethe ansatz, transfer matrix, Yangian highest-weight states, etc. Indeed, we start by establishing the precise connection between the $\mathrm{su}(m) \mathrm{KY}$ model and the $\mathrm{su}(1 \mid m)$ 
supersymmetric HS spin chain with a suitable chemical potential term for arbitrary $m$, thus generalizing the well-known result for $m=2$. Since the partition function of the latter chain was recently evaluated in Ref. [22] by using Polychronakos's freezing trick, this immediately yields a novel closed formula for the partition function of the $\mathrm{su}(m)$ KY model. A remarkable property of this partition function is that it can be recast as the partition function of an equivalent (inhomogeneous) vertex model with simple interactions [23]. We show that both partition functions are polynomials in appropriate variables, whose coefficients are nothing but the corresponding restricted partition functions on subspaces with well-defined magnon content. This crucial observation provides a closed formula for the restricted partition function of the $\mathrm{su}(m) \mathrm{KY}$ model (in the presence of an external magnetic field and a chemical potential term) on each of these subspaces. Finally, by analyzing the restricted partition function of the equivalent vertex model we are able to express the spectrum of the $\mathrm{su}(m) \mathrm{KY}$ model in each subspace with well-defined magnon content in terms of suitably restricted supersymmetric Haldane motifs and their corresponding Young tableaux. This yields a complete and rigorous description of the spectrum in the latter subspaces, including a systematic way for determining the degeneracy of each level, which implies the Saiga-Kuramoto conjecture as a particular case.

It should be noted that, while the traditional freezing trick allows one to compute the partition function (and, in principle, the spectrum) of certain integrable systems, it does not provide any information about the corresponding wave functions. On the other hand, the analysis of the spectrum of the $\operatorname{su}(m) \mathrm{KY}$ model by the method described above, extends the freezing trick and, more importantly, the equivalence to a vertex model, to subspaces of the Hilbert space with well-defined magnon content. It is in fact this connection with a restricted vertex model what makes it possible to identify all energy eigenvalues within any subspace of the Hilbert space with well-defined magnon content. In other words, our approach not only yields the complete spectrum of the $\mathrm{su}(m) \mathrm{KY}$ model but also the magnon numbers or spin content of the corresponding wave functions.

Our approach yields several additional results that we shall now briefly discuss. In the first place, as a consequence of the general discussion of the equivalence of the $\mathrm{su}(\mathrm{m})$ $\mathrm{KY}$ model to an $\mathrm{su}(1 \mid m)$ supersymmetric HS chain with a suitable chemical potential term, we construct a new model of KY type with general elliptic interactions which can be mapped to a corresponding $\mathrm{su}(1 \mid m)$ generalization of Inozemtsev's chain [24]. This model certainly deserves further study, since it smoothly interpolates between the standard (nearest-neighbors) $t-J$ model and the (long-range) KY model. Secondly, as an application of the description of the spectrum of the KY model in terms of supersymmetric Young tableaux, we determine the ground state of the spin 1/2 model in an external magnetic field in the thermodynamic limit. In particular, we give a complete description of the different ground state phases, characterized by their spin content i.e., $\mathrm{su}(1 \mid 2), \mathrm{su}(1 \mid 1)$ and $\mathrm{su}(0 \mid 2)$, apart from the trivial phases consisting of only holes or fermions of one type - in terms of the magnetic field strength and the charge chemical 
potential. This description goes beyond previously known results, which are restricted to the genuinely $\mathrm{su}(1 \mid 2)$ phase. In particular, we show that the strong spin-charge separation characteristic of the long-range $t$ - $J$ model at low temperatures [25] occurs in all nontrivial phases.

The paper is organized as follows. In Section 2 we introduce the model and show its equivalence to a supersymmetric HS chain with suitable chemical potential terms. We also introduce the model's elliptic version and discuss its connection with the supersymmetric extension of Inozmetsev's elliptic chain. The model's partition function is then computed in Section 3 by exploiting its equivalence to a supersymmetric HS chain. In Section 4 we recast the partition function as that of a suitable inhomogeneous vertex model and derive the model's restricted partition function on subspaces with well-defined magnon content. As explained above, this yields an explicit and complete description of the spectrum on the latter subspaces, including each level's degeneracy, in terms of suitable supersymmetric Young tableaux. Section 5 is devoted to a complete analysis of the ground state phases of the spin $1 / 2$ model in an external magnetic field in the thermodynamic limit. In Section 6 we present our conclusions and outline several future developments. The paper ends with a short technical appendix in which we present the proof of a new result regarding the degeneracy of reverse motifs.

\section{The models}

We shall deal in this paper with a class of $\operatorname{su}(m) t-J$ type models, consisting of a onedimensional lattice with $N$ sites each of which can be either empty or occupied by a single fermion with $m$ internal degrees of freedom. We shall be mainly interested in long-ranged models of the latter type, in which the spin and charge interactions among the fermions and their hopping amplitude involve all possible pairs of lattice sites. More precisely, we shall take as the model's Hamiltonian

$$
H_{0}=\sum_{1 \leqslant i<j \leqslant N} \mathcal{P}\left\{-t_{i j} \sum_{\sigma=1}^{m}\left(c_{i \sigma}^{\dagger} c_{j \sigma}+c_{j \sigma}^{\dagger} c_{i \sigma}\right)+2 J_{i j} \mathbf{T}_{i} \cdot \mathbf{T}_{j}-2 V_{i j} n_{i} n_{j}\right\} \mathcal{P} \equiv \sum_{1 \leqslant i<j \leqslant N} H_{i j}
$$

or equivalently

$$
H_{0}=\sum_{i \neq j} \mathcal{P}\left\{-t_{i j} \sum_{\sigma} c_{i \sigma}^{\dagger} c_{j \sigma}+J_{i j} \mathbf{T}_{i} \cdot \mathbf{T}_{j}-V_{i j} n_{i} n_{j}\right\} \mathcal{P},
$$

where $t_{i j}=t_{j i}, J_{i j}=J_{j i}, V_{i j}=V_{j i}$ are real constants. We shall also assume that the model (2.1)-(2.2) is translation-invariant, i.e., that

$$
t_{i j}=t(i-j), \quad J_{i j}=J(i-j), \quad V_{i j}=V(i-j)
$$

with

$$
t(x)=t(-x)=t(N-x),
$$

$\ddagger$ Here and in what follows, unless otherwise stated sums and products over Latin indexes run over the set $1, \ldots, N$, while Greek indices range from 1 to $m$. 
and similarly for $J(x), V(x)$. In the latter equations $c_{i \sigma}^{\dagger}$ (respectively $c_{i \sigma}$ ) denotes the operator creating (resp. destroying) a fermion of type $\sigma$ at site $i$ and $n_{i}=\sum_{\sigma} n_{i \sigma}$, where $n_{i \sigma}=c_{i \sigma}^{\dagger} c_{i \sigma}$, is the total number of fermions at site $i$. The operator $\mathcal{P}$ is the projector onto single-occupancy states, in which each site is occupied by at most one fermion. Finally, $\mathbf{T}_{i} \equiv\left(T_{i}^{1}, \ldots, T_{i}^{m^{2}-1}\right)$, where $T_{i}^{r}$ is the $r$-th su $(m)$ Hermitian generator in the fundamental representation acting on the $i$-th site (with a suitable normalization that we shall specify below). Thus the first term (proportional to $t_{i j}$ ) in Eqs. (2.1)-(2.2) accounts for the hopping of fermions between sites $i$ and $j$, while the last two terms respectively model the spin (exchange) and charge interaction between the latter sites.

The Hamiltonian (2.1)-(2.2) encompasses several well-known models, which we shall briefly review. To begin with, note that a nearest-neighbors version of the Hamiltonian (2.1)-(2.2) is obtained by taking $t(x), J(x), V(x)$ proportional to the $N$ periodic extension of

$$
\delta_{1, x}+\delta_{N-1, x}, \quad 1 \leqslant x \leqslant N-1 .
$$

When $m=2$, this is the original $t$ - $J$ model introduced in Ref. [1]. On the other hand, the long-ranged supersymmetric Kuramoto-Yokoyama model [9,10] follows from Eqs. (2.2)-(2.3) when

$$
t(x)=J(x)=4 V(x)=\frac{t \pi^{2}}{N^{2}} \sin ^{-2}(\pi x / N),
$$

where $t$ is a positive real parameter. More generally, when $m=2$ the model (2.2)-(2.3) is supersymmetric provided that $t(x)=J(x)=4 V(x)$. In fact, one of our aims in this section is to generalize the latter result to the $\mathrm{su}(m)$ case with arbitrary $m>2$. As we shall next see, the key idea in this respect is to map the original Hamiltonian (2.1) to that of a suitable supersymmetric spin chain in which the holes are regarded as bosons.

More precisely, consider a one-dimensional lattice (spin chain) each of whose sites are occupied either by a boson or an $\mathrm{su}(m)$ fermion. Thus the model's Hilbert space is $\hat{\mathcal{H}}=\otimes_{i=1}^{N} \hat{\mathcal{H}}_{i}$, where $\hat{\mathcal{H}}_{i}$ is the linear span of the one-particle states $b_{i}^{\dagger}|\hat{\Omega}\rangle_{i}, f_{i \sigma}^{\dagger}|\hat{\Omega}\rangle_{i}$ with $\sigma=1, \ldots, m, b_{i}^{\dagger}, f_{i \sigma}^{\dagger}$ are the operators creating respectively a boson and a fermion of type $\sigma$ at the $i$-th site, and $|\hat{\Omega}\rangle_{i}$ is the vacuum. Similarly, denote by $\mathcal{H}=\otimes_{i=1}^{N} \mathcal{H}_{i}$ the Hilbert space of the original model (2.1), where $\mathcal{H}_{i}$ is spanned by the vacuum $|\Omega\rangle_{i}$ and the one-particle states $c_{i \sigma}^{\dagger}|\Omega\rangle_{i}$. We now introduce the unitary mapping $\varphi: \mathcal{H} \rightarrow \hat{\mathcal{H}}$ defined by

$$
\varphi|\Omega\rangle_{i}=b_{i}^{\dagger}|\hat{\Omega}\rangle_{i}, \quad \varphi\left(c_{i \sigma}^{\dagger}|\Omega\rangle_{i}\right)=f_{i \sigma}^{\dagger}|\hat{\Omega}\rangle_{i}
$$

This mapping induces a natural way of associating to each linear operator $A$ acting on $\mathcal{H}$ a corresponding linear operator $\hat{A}=\varphi A \varphi^{-1}=\varphi A \varphi^{\dagger}$ acting on $\hat{\mathcal{H}}$. Note, in particular, that $\left(A^{\dagger} \hat{)}=\varphi A^{\dagger} \varphi^{\dagger} \equiv \hat{A}^{\dagger}\right.$. It is straightforward to check that under this correspondence $\hat{c}_{i \sigma}=b_{i}^{\dagger} f_{i \sigma}$, since both operators agree on the canonical basis of $\hat{\mathcal{H}}_{i}$. Note than on $\mathcal{H}_{i}$ we have $c_{i \sigma}=\mathcal{P} c_{i \sigma} \mathcal{P}$, so that we can also write

$$
\left(\mathcal{P} c_{i \sigma} \mathcal{P}\right)=b_{i}^{\dagger} f_{i \sigma} \equiv X_{i}^{0 \sigma}
$$


and therefore (since $\mathcal{P}$ is Hermitian, being a projector)

$$
\left(\mathcal{P} c_{i \sigma}^{\dagger} \mathcal{P}\right) \hat{c}_{i \sigma}^{\dagger}=f_{i \sigma}^{\dagger} b_{i} \equiv X_{i}^{\sigma 0} \text {. }
$$

We shall also need in the sequel the relations

$$
\begin{aligned}
& \left(\mathcal{P} c_{i \sigma}^{\dagger} c_{i \sigma^{\prime}} \mathcal{P}\right) \hat{)}=f_{i \sigma}^{\dagger} f_{i \sigma^{\prime}} \equiv X_{i}^{\sigma \sigma^{\prime}}, \\
& {\left[\mathcal{P}\left(1-n_{i}\right) \mathcal{P}\right]=b_{i}^{\dagger} b_{i} \equiv X_{i}^{00},}
\end{aligned}
$$

which easily follow from the previous ones. For instance, taking into account that $c_{i \sigma^{\prime}} \mathcal{P}=\mathcal{P} c_{i \sigma^{\prime}} \mathcal{P}$ and $\mathcal{P}^{2}=\mathcal{P}$ we have

$$
\begin{aligned}
\left(\mathcal{P} c_{i \sigma}^{\dagger} c_{i \sigma^{\prime}} \mathcal{P}\right) & =\left(\mathcal{P} c_{i \sigma}^{\dagger} \mathcal{P} \cdot \mathcal{P} c_{i \sigma^{\prime}} \mathcal{P}\right) \\
& =f_{i \sigma}^{\dagger} b_{i} b_{i}^{\dagger} f_{i \sigma^{\prime}}=X_{i}^{\sigma \sigma^{\prime}}+f_{i \sigma}^{\dagger} b_{i}^{\dagger} f_{i \sigma} b_{i}=X_{i}^{\sigma \sigma^{\prime}},
\end{aligned}
$$

since $f_{i \sigma} b_{i}=0$ on $\hat{\mathcal{H}}_{i}$.

Consider next the $\operatorname{su}(1 \mid m)$ supersymmetric permutation operators $P_{i j}^{(1 \mid m)}: \hat{\mathcal{H}} \rightarrow \hat{\mathcal{H}}$ (with $i<j$ ), whose action on the canonical basis

$$
\left|\sigma_{1} \cdots \sigma_{N}\right\rangle \equiv a_{1 \sigma_{1}}^{\dagger} \cdots a_{N \sigma_{N}}^{\dagger}|\hat{\Omega}\rangle, \quad \sigma_{1}, \ldots, \sigma_{N} \in\{0, \ldots, m\}
$$

where $a_{i 0}=b_{i}$ and $a_{i \sigma}=f_{i \sigma}$ for $\sigma \geqslant 1$ and $|\hat{\Omega}\rangle=\otimes_{i}|\hat{\Omega}\rangle_{i}$ is the global vacuum, is given by $[26]$

$$
P_{i j}^{(1 \mid m)}\left|\cdots \sigma_{i} \cdots \sigma_{j} \cdots\right\rangle=\epsilon(\boldsymbol{\sigma})\left|\cdots \sigma_{j} \cdots \sigma_{i} \cdots\right\rangle .
$$

The sign $\epsilon(\boldsymbol{\sigma})$ is 1 (respectively -1 ) if $\sigma_{i}=\sigma_{j}=0$ (resp. $\sigma_{i}, \sigma_{j} \geqslant 1$ ), while for $\sigma_{i} \sigma_{j}=0$ and $\sigma_{i} \neq \sigma_{j}$ it is equal to the number of fermionic spins $\sigma_{k}$ with $i+1 \leqslant k \leqslant j-1$. It is well known $[19,26,27]$ that $P_{i j}^{(1 \mid m)}$ can be expressed in terms of creation and annihilation operators as follows:

$$
P_{i j}^{(1 \mid m)}=\sum_{\alpha, \beta=0}^{m} a_{i \alpha}^{\dagger} a_{j \beta}^{\dagger} a_{i \beta} a_{j \alpha}=\sum_{\alpha, \beta=0}^{m}(-1)^{p(\beta)} X_{i}^{\alpha \beta} X_{j}^{\beta \alpha},
$$

where $p(0)=0$ and $p(\sigma)=1$ for $\sigma \geqslant 1$. We thus have

$$
P_{i j}^{(1 \mid m)}=X_{i}^{00} X_{j}^{00}+\sum_{\sigma}\left(X_{i}^{\sigma 0} X_{j}^{0 \sigma}-X_{i}^{0 \sigma} X_{j}^{\sigma 0}\right)-P_{i j}
$$

where

$$
P_{i j}=\sum_{\sigma, \sigma^{\prime}} X_{i}^{\sigma \sigma^{\prime}} X_{j}^{\sigma^{\prime} \sigma}
$$

is the ordinary permutation operator when acting on purely fermionic states.

Our next goal is to relate the product $\mathbf{T}_{i} \cdot \mathbf{T}_{j}$ appearing in the Hamiltonian (2.1) with the supersymmetric permutation operator $P_{i j}^{(1 \mid m)}$. To this end, note first of all that the components $T_{i}^{r}$ of $\mathbf{T}_{i}$ are defined in the usual way as

$$
T_{i}^{r}=\sum_{\sigma, \sigma^{\prime}} T_{\sigma \sigma^{\prime}}^{r} c_{i \sigma}^{\dagger} c_{i \sigma^{\prime}}
$$

where the complex numbers $T_{\sigma \sigma^{\prime}}^{r}$ are the matrix elements of the $r$-th (Hermitian) generator of $\mathrm{su}(m)$ in the fundamental representation. We shall normalize the $m \times m$ matrices $T^{r} \equiv\left(T_{\sigma \sigma^{\prime}}^{r}\right)_{1 \leqslant \sigma, \sigma^{\prime} \leqslant m}$ so that

$$
\operatorname{tr}\left(T^{r} T^{s}\right)=\frac{1}{2} \delta_{r s} .
$$


In particular, when $m=2$ the operator $T^{r}$ can be taken as the usual spin $1 / 2$ operator $S^{r}=\sigma^{r} / 2$, where $\sigma^{r}$ is the $r$-th Pauli matrix. In order to relate $\mathbf{T}_{i} \cdot \mathbf{T}_{j}$ with $P_{i j}^{(1 \mid m)}$ we shall make use of the identity

$$
2 \sum_{r=1}^{m^{2}-1}\left(T^{r}\right)_{\sigma \sigma^{\prime}}\left(T^{r}\right)_{\mu \mu^{\prime}}=\delta_{\sigma \mu^{\prime}} \delta_{\sigma^{\prime} \mu}-\frac{1}{m} \delta_{\sigma \sigma^{\prime}} \delta_{\mu \mu^{\prime}}
$$

which is a direct consequence of the completeness of the generators $T^{r}$ together with the identity matrix. From Eqs. (2.8), (2.11) and (2.12) we obtain

$$
\begin{aligned}
2\left(\mathcal{P} \mathbf{T}_{i} \cdot \mathbf{T}_{j} \mathcal{P}\right) & =2 \sum_{r=1}^{m^{2}-1} \sum_{\substack{\sigma, \sigma^{\prime} \\
\mu, \mu^{\prime}}} T_{\sigma \sigma^{\prime}}^{r} T_{\mu \mu^{\prime}}^{r} X_{i}^{\sigma \sigma^{\prime}} X_{j}^{\mu \mu^{\prime}}=\sum_{\sigma, \sigma^{\prime}} X_{i}^{\sigma \sigma^{\prime}} X_{j}^{\sigma^{\prime} \sigma}-\frac{1}{m} \sum_{\sigma, \mu} X_{i}^{\sigma \sigma} X_{i}^{\mu \mu} \\
& =P_{i j}-\frac{1}{m} \hat{n}_{i} \hat{n}_{j},
\end{aligned}
$$

where $\hat{n}_{k}=\sum_{\sigma} f_{k \sigma}^{\dagger} f_{k \sigma}$ denotes the total number of fermions (created by the operators $f_{k \sigma}^{\dagger}$ ) at the $k$-th site. From Eq. (2.10) for the supersymmetric permutation operator $P_{i j}^{(1 \mid m)}$ we obtain, after some algebra,

$$
P_{i j}^{(1 \mid m)}+\hat{n}_{i}+\hat{n}_{j}-1=\hat{A}_{i j}
$$

where

$$
A_{i j}=\mathcal{P}\left[\sum_{\sigma}\left(c_{i \sigma}^{\dagger} c_{j \sigma}+c_{j \sigma}^{\dagger} c_{i \sigma}\right)-2 \mathbf{T}_{i} \cdot \mathbf{T}_{j}+\left(1-\frac{1}{m}\right) n_{i} n_{j}\right] \mathcal{P}
$$

Comparing with Eq. (2.1) we deduce that $H_{i j}$ will be proportional to $A_{i j}$ provided that

$$
t(x)=J(x)=2\left(1-\frac{1}{m}\right)^{-1} V(x),
$$

and in that case

$$
\hat{H}_{i j}=t_{i j}\left(1-P_{i j}^{(1 \mid m)}-\hat{n}_{i}-\hat{n}_{j}\right) .
$$

In other words, when condition (2.15) is satisfied, i.e., when $H_{0}$ is of the form

$$
H_{0}=\sum_{i<j} t_{i j} \mathcal{P}\left[-\sum_{\sigma}\left(c_{i \sigma}^{\dagger} c_{j \sigma}+c_{j \sigma}^{\dagger} c_{i \sigma}\right)+2 \mathbf{T}_{i} \cdot \mathbf{T}_{j}-\left(1-\frac{1}{m}\right) n_{i} n_{j}\right] \mathcal{P},
$$

the corresponding Hamiltonian $\hat{H}_{0}$ is given by

$$
\hat{H}_{0}=\sum_{i<j} t_{i j}\left(1-P_{i j}^{(1 \mid m)}-\hat{n}_{i}-\hat{n}_{j}\right)=\sum_{i<j} t_{i j}\left(1-P_{i j}^{(1 \mid m)}\right)-\sum_{i \neq j} t_{i j} \hat{n}_{j} .
$$

Note that so far we have not used the translation-invariance conditions (2.3)-(2.4), so that the previous result is valid in full generality. On the other hand, when the model (2.1) is translation-invariant we can use Eqs. (2.3)-(2.4) to further simplify the last term in the previous equation. Indeed, in this case

$$
\sum_{i \neq j} t_{i j} \hat{n}_{j}=\sum_{j}\left(\sum_{i ; i \neq j} t_{i j}\right) \hat{n}_{j}
$$

with

$$
\sum_{i ; i \neq j} t_{i j}=\sum_{k=1-j}^{-1} t(k)+\sum_{k=1}^{N-j} t(k)=\sum_{k=N-j+1}^{N-1} t(k-N)+\sum_{k=1}^{N-j} t(k)=\sum_{k=1}^{N-1} t(k) \equiv t_{0},
$$


so that

$$
\hat{H}_{0}=\sum_{i<j} t_{i j}\left(1-P_{i j}^{(1 \mid m)}\right)-t_{0} \mathcal{F}
$$

where

$$
\mathcal{F} \equiv \sum_{i} \hat{n}_{i}
$$

is the total number of fermions.

Summarizing, we have shown that the translation-invariant $\mathrm{su}(m)$ model (2.1)-(2.3) is supersymmetric if its coefficients are related by Eq. (2.15). When this is the case this general model reduces to (2.16), which is equivalent to the $\mathrm{su}(1 \mid m)$ supersymmetric spin chain (2.17). It should be stressed that the coefficient of the charge interaction term $n_{i} n_{j}$ in the supersymmetric $t-J$ Hamiltonian (2.16) must depend on $m$ as specified in the latter equation, a fact that does not seem to have been previously noted in the literature.

Note that Eqs. (2.16)-(2.17) are also valid for $m=1$. In this case the Hamiltonian (2.16) is that of a free fermion system (the terms proportional to $\mathbf{T}_{i} \cdot \mathbf{T}_{j}$ and $n_{i} n_{j}$ vanish identically), as first noted by Göhmann and Wadati [28]. This fact was recently exploited in Refs. $[29,30]$ to evaluate the entanglement entropy of the ground state of (translation-invariant) $\mathrm{su}(1 \mid 1)$ spin chains of HS type.

In the rest of this work we shall be mainly concerned with the supersymmetric $t-J$ model (2.16) with

$$
t(x)=t(\pi / N)^{2} \sin ^{-2}(\pi x / N)
$$

which is the $\mathrm{su}(m)$ version of the original KY model. In this case [31,32]

$$
\sum_{i \neq j} \sin ^{-2}(\pi(i-j) / N)=\frac{N}{3}\left(N^{2}-1\right)
$$

so that

$$
t_{0}=\frac{t \pi^{2}}{3 N^{2}}\left(N^{2}-1\right)
$$

Hence the Hamiltonian $\hat{H}_{0}$ of the equivalent $\mathrm{su}(1 \mid m)$ supersymmetric spin chain can be written as

$$
\hat{H}_{0}=\frac{2 t \pi^{2}}{N^{2}}\left[H_{\mathrm{HS}}^{(1 \mid m)}-\frac{1}{6}\left(N^{2}-1\right) \mathcal{F}\right]
$$

where

$$
H_{\mathrm{HS}}^{(1 \mid m)}=\frac{1}{2} \sum_{i<j} \frac{1-P_{i j}^{(1 \mid m)}}{\sin ^{2}(\pi(i-j) / N)}
$$

is the Hamiltonian of the $\operatorname{su}(1 \mid m)$ Haldane-Shastry spin chain $[11,12,19,33]$. In fact, the model (2.21) was introduced by Kawakami in the early 90's [15]. On the other hand, when $t(x)$ is proportional to Eq. (2.5) the Hamiltonian (2.16) reduces to the $\mathrm{su}(m)$ version of the original (nearest-neighbor) $t-J$ model

$H_{0}=t \sum_{i} \mathcal{P}\left[-\sum_{\sigma}\left(c_{i \sigma}^{\dagger} c_{i+1, \sigma}+c_{i+1, \sigma}^{\dagger} c_{i \sigma}\right)+2 \mathbf{T}_{i} \cdot \mathbf{T}_{i+1}-\left(1-\frac{1}{m}\right) n_{i} n_{i+1}\right] \mathcal{P}$ 
where $N+1 \equiv 1$. The equivalent $\mathrm{su}(1 \mid \mathrm{m})$ supersymmetric chain Hamiltonian is given by

$$
\hat{H}_{0}=t \sum_{i}\left(1-P_{i, i+1}^{(1 \mid m)}\right)-2 t \mathcal{F},
$$

where $P_{N, N+1}^{(1 \mid m)} \equiv P_{1 N}^{(1 \mid m)}$, which is essentially the $\mathrm{su}(1 \mid m)$ Uimin-Lai-Sutherland model $[4,34,35]$.

Next, inspired by Inozemtsev's elliptic spin chain [24], we introduce a one-parameter family of supersymmetric su $(m) t-J$ models (2.16) which smoothly interpolate between the $\mathrm{su}(m) \mathrm{KY}$ model (2.16)-(2.18) and the (periodic) nearest-neighbors $\mathrm{su}(m) t-J$ model (2.23). More precisely, let

$$
t(x)=t\left(\frac{\alpha}{\pi}\right)^{2} \sinh ^{2}(\pi / \alpha)\left(\wp_{N}(x)-\frac{2 \tilde{\eta}_{1}}{\alpha^{2}}\right),
$$

where

$$
\wp_{N}(x) \equiv \wp(x ; N / 2, \mathrm{i} \alpha / 2), \quad \tilde{\eta}_{1} \equiv \zeta(1 / 2 ; 1 / 2, \mathrm{i} N /(2 \alpha))
$$

and $\alpha>0$. In the latter formulas $\wp\left(x ; \omega_{1}, \omega_{3}\right)$ and $\zeta\left(x ; \omega_{1}, \omega_{3}\right)$ denote respectively the Weierstrass elliptic function with half-periods $\omega_{1}, \omega_{3}$ and its corresponding zeta function, defined by

$$
\begin{aligned}
& \zeta\left(z ; \omega_{1}, \omega_{3}\right)=\frac{1}{z}+\sum_{l, n \in \mathbb{Z}^{2}-\{(0,0)\}}\left[\frac{1}{z-2 l \omega_{1}-2 n \omega_{3}}+\frac{z}{\left(2 l \omega_{1}+2 n \omega_{3}\right)^{2}}\right], \\
& \wp\left(z ; \omega_{1}, \omega_{3}\right)=-\zeta^{\prime}\left(z ; \omega_{1}, \omega_{3}\right) .
\end{aligned}
$$

It can be shown $[36,37]$ that when $1 \leqslant x \leqslant N-1$

$$
\lim _{\alpha \rightarrow 0+} t(x)=t\left(\delta_{1, x}+\delta_{-1, x}\right),
$$

while the $\alpha \rightarrow+\infty$ limit of Eq. (2.25) is Eq. (2.18). Note that the constant $t_{0}$ for the function (2.25) is given by

$$
t_{0}=\frac{2 t}{\pi^{2}} \sinh ^{2}(\pi / \alpha)\left(\tilde{\eta}_{1}-\eta_{1}\right), \quad \eta_{1} \equiv \zeta(1 / 2 ; 1 / 2, \mathrm{i} /(2 \alpha))
$$

(see, e.g., Ref. [38]). Thus the Hamiltonian (2.17) of the $\mathrm{su}(1 \mid m)$ supersymmetric spin chain equivalent to the $\mathrm{su}(m) t-J$ model (2.16) with elliptic interactions (2.25) is given by

$$
\hat{H}_{0}=t H_{I}^{(1 \mid m)}-\frac{2 t}{\pi^{2}} \sinh ^{2}(\pi / \alpha)\left(\tilde{\eta}_{1}-\eta_{1}\right) \mathcal{F}
$$

where

$$
H_{I}^{(1 \mid m)}=\left(\frac{\alpha}{\pi}\right)^{2} \sinh ^{2}(\pi / \alpha) \sum_{i<j}\left(\wp_{N}(i-j)-\frac{2 \tilde{\eta}_{1}}{\alpha^{2}}\right)\left(1-P_{i j}^{(1 \mid m)}\right)
$$

is the $\mathrm{su}(1 \mid m)$ version of Inozemtsev's elliptic spin chain [24]. In fact, for $m=1$ the partition function and thermodynamics of the latter chain were derived in Ref. [37], and the entanglement entropy of its ground state was analyzed in Ref. [29]. 


\section{Partition function}

In this section we shall compute in closed form the partition function of the $\mathrm{su}(m)$ KY model (2.16)-(2.18) by exploiting its equivalence with the $\mathrm{su}(1 \mid m)$ spin chain Hamiltonian of Haldane-Shastry type (2.21)-(2.22). As a matter of fact, we shall consider the more general Hamiltonian

$$
H=H_{0}-\frac{1}{2} \sum_{\sigma=1}^{m-1} h_{\sigma}\left(n^{\sigma}-n^{m}\right)-\mu_{c} \sum_{\sigma} n^{\sigma} \equiv H_{0}+H_{1},
$$

where $H_{0}$ is given by Eqs. (2.16)-(2.18) and

$$
n^{\sigma} \equiv \sum_{i} n_{i \sigma}
$$

denotes the total number of fermions of type $\sigma$. The last term in $H_{1}$ is the chemical potential of the fermions (or, equivalently, of the total electric charge), while the first one has a natural interpretation as arising from the interaction with an external $\mathrm{su}(m)$ magnetic field with strengths $h_{1}, \ldots, h_{m-1}$. Indeed, for $m=2$ the term $-\left(h_{1} / 2\right)\left(n^{1}-n^{2}\right)$ equals $-h_{1} S^{z}$, where $S^{z}$ is the $z$ component of the total spin operator. This is indeed the contribution to the energy arising from the interaction with the magnetic field $h_{1} \mathbf{e}_{z}$ of a charged fermion (with gyromagnetic ratio $g=2$, and unit mass and electric charge in natural units). More generally, for arbitrary $m \geqslant 2$ we have

$$
n^{\sigma}-n^{m}=\sum_{i}\left(n_{i \sigma}-n_{i m}\right)
$$

where the operators $\mathrm{i}\left(n_{k \sigma}-n_{k m}\right)$ generate the standard $\mathrm{su}(m)$ Cartan subalgebra at the $k$-th site. By Eq. (2.8), the $\operatorname{su}(1 \mid m)$ spin chain Hamiltonian $\hat{H}$ equivalent to $H$ is $\hat{H}=\hat{H}_{0}+\hat{H}_{1}$, where

$$
\hat{H}_{1}=-\frac{1}{2} \sum_{\sigma=1}^{m-1} h_{\sigma}\left(\mathcal{N}_{\sigma}-\mathcal{N}_{m}\right)-\mu_{c} \mathcal{F}
$$

and $\mathcal{N}_{\sigma} \equiv \sum_{i} \hat{n}^{\sigma}$ is the total numbers of fermions (created by $f_{i \sigma}^{\dagger} f_{i \sigma}$ ) of type $\sigma$. More explicitly, we have

$$
\hat{H}=J H_{\mathrm{HS}}^{(1 \mid m)}-\frac{1}{2} \sum_{\sigma=1}^{m-1} h_{\sigma}\left(\mathcal{N}_{\sigma}-\mathcal{N}_{m}\right)-\left(t_{0}+\mu_{c}\right) \mathcal{F},
$$

with $t_{0}$ given by Eq. (2.20) and $J=2 t \pi^{2} / N^{2}$. The latter equation can be more concisely rewritten as

$$
\hat{H}=J H_{\mathrm{HS}}^{(1 \mid m)}-\sum_{\sigma} \mu_{\sigma} \mathcal{N}_{\sigma}
$$

where $\mu_{\sigma}$ is the chemical potential of the fermion of type $\sigma$, given by

$$
\begin{aligned}
& \mu_{\sigma}=\frac{1}{2} h_{\sigma}+\mu_{c}+t_{0}, \quad 1 \leqslant \sigma \leqslant m-1 ; \\
& \mu_{m}=-\frac{1}{2} \sum_{\sigma=1}^{m-1} h_{\sigma}+\mu_{c}+t_{0} .
\end{aligned}
$$


From the above remarks, it follows that the partition function $\mathcal{Z}$ of the supersymmetric $\mathrm{su}(m) \mathrm{KY}$ model (3.1) coincides with that of the $\mathrm{su}(1 \mid m)$ supersymmetric HaldaneShastry chain with a chemical potential term given in Eq. (3.3). The partition function of the latter model has been recently evaluated in Ref. [22] by taking advantage of its connection with the supersymmetric spin Sutherland model via Polychronakos's freezing trick [39, 40], with the result

$$
\mathcal{Z}(q ; \boldsymbol{\mu})=\sum_{\mathbf{k} \in \mathcal{P}_{N}} d(\mathbf{k}) q^{\sum_{i=1}^{r-1} J K_{i}\left(N-K_{i}\right)} \prod_{i=1}^{N-r}\left(1-q^{J K_{i}^{\prime}\left(N-K_{i}^{\prime}\right)}\right)
$$

Here $q \equiv \mathrm{e}^{-1 / T}, \mathbf{k}=\left(k_{1}, \ldots, k_{r}\right) \in(\mathbb{N} \cup\{0\})^{r}, \mathcal{P}_{N}$ is the set of partitions (with order taken into account) of the integer $N, K_{i} \equiv \sum_{j=1}^{i} k_{j}$, and the $N-r$ positive integers $K_{i}^{\prime}$ are defined by

$$
\left\{K_{1}^{\prime}, \ldots, K_{N-r}^{\prime}\right\}=\{1, \ldots, N-1\}-\left\{K_{1}, \ldots, K_{r-1}\right\}
$$

For each multiindex $\mathbf{k} \in(\mathbb{N} \cup\{0\})^{r}$, the coefficient $d(\mathbf{k})$ is defined by

$$
d(\mathbf{k})=\prod_{i=1}^{r} \sum_{j=0}^{\min \left(k_{i}, m\right)} e_{j}\left(q^{-\boldsymbol{\mu}}\right), \quad q^{-\boldsymbol{\mu}} \equiv\left(q^{-\mu_{1}}, \ldots, q^{-\mu_{m}}\right),
$$

where

$$
e_{j}\left(y_{1}, \ldots, y_{m}\right) \equiv \sum_{1 \leqslant i_{1}<\cdots<i_{j} \leqslant m} y_{i_{1}} \cdots y_{i_{j}}
$$

denotes the elementary symmetric polynomial of degree $j \leqslant m$ in $m$ variables $y_{1}, \ldots, y_{m}$ (with $e_{0} \equiv 1$ ). By the above remarks, Eq. (3.6) yields also the partition function of the supersymmetric $\mathrm{su}(m) \mathrm{KY}$ model (3.1). This is one of the main results of the present work.

Equation (3.7) for $d(\mathbf{k})$ can be considerably simplified introducing the numbers $\nu_{i}(\mathbf{k})$ defined by

$$
\begin{aligned}
& \nu_{l}(\mathbf{k})=\left|\left\{i \in\{1, \ldots, r\}: k_{i}=l\right\}\right|, \quad l=1, \ldots, m-1, \\
& \nu_{m}(\mathbf{k})=\left|\left\{i \in\{1, \ldots, r\}: k_{i} \geqslant m\right\}\right|,
\end{aligned}
$$

where $|A|$ denotes the cardinal of the set $A$. We then have

$$
d(\mathbf{k})=\prod_{l=1}^{m-1}\left[\sum_{j=0}^{l} e_{j}\left(q^{-\mu}\right)\right]^{\nu_{l}(\mathbf{k})} \cdot \prod_{j=1}^{m}\left(1+q^{-\mu_{j}}\right)^{\nu_{m}(\mathbf{k})} .
$$

For instance, in the $\mathrm{su}(2)$ and $\mathrm{su}(3)$ cases we respectively have

$$
d(\mathbf{k})=\left(1+q^{-\mu_{1}}+q^{-\mu_{2}}\right)^{\nu_{1}(\mathbf{k})}\left[\left(1+q^{-\mu_{1}}\right)\left(1+q^{-\mu_{2}}\right)\right]^{\nu_{2}(\mathbf{k})}
$$

and

$$
d(\mathbf{k})=\left(1+\sum_{\sigma} q^{-\mu_{\sigma}}\right)^{\nu_{1}(\mathbf{k})}\left(1+\sum_{\sigma} q^{-\mu_{\sigma}}+\sum_{\sigma<\sigma^{\prime}} q^{-\left(\mu_{\sigma}+\mu_{\sigma^{\prime}}\right)}\right)^{\nu_{2}(\mathbf{k})} \prod_{\sigma}\left(1+q^{-\mu_{\sigma}}\right)^{\nu_{3}(\mathbf{k})} .
$$


The latter expressions becomes even simpler when applied to the "pure" supersymmetric KY model (i.e., without magnetic field or chemical potential terms), for which $\mu_{\sigma}=t_{0}$ for all $\sigma=1, \ldots, m$ according to Eqs. (3.4)-(3.5). Indeed, in this case we have

$$
e_{j}\left(q^{-\mu}\right)=e_{j}\left(q^{-t_{0}}, \ldots, q^{-t_{0}}\right)=\sum_{1 \leqslant i_{1}<\cdots<i_{j} \leqslant m} q^{-j t_{0}}=\left(\begin{array}{c}
m \\
j
\end{array}\right) q^{-j t_{0}}
$$

and hence

$$
d(\mathbf{k})=\left(1+q^{-t_{0}}\right)^{m \nu_{m}(\mathbf{k})} \prod_{l=1}^{m-1}\left[\sum_{j=0}^{l}\left(\begin{array}{c}
m \\
j
\end{array}\right) q^{-j t_{0}}\right]^{\nu_{l}(\mathbf{k})} .
$$

For instance, the partition function of the original $(\mathrm{su}(2))$ supersymmetric KY model is given by

$$
\mathcal{Z}_{N}(q)=\sum_{\mathbf{k} \in \mathcal{P}_{N}}\left(1+2 q^{-t_{0}}\right)^{\nu_{1}(\mathbf{k})}\left(1+q^{-t_{0}}\right)^{2 \nu_{2}(\mathbf{k})} q^{\sum_{i=1}^{r-1} J K_{i}\left(N-K_{i}\right)} \prod_{i=1}^{N-r}\left(1-q^{J K_{i}^{\prime}\left(N-K_{i}^{\prime}\right)}\right) .
$$

\section{Spectrum and motifs}

In this section we shall give a complete description of the spectrum of the supersymmetric $\mathrm{su}(m) \mathrm{KY}$ model (2.16)-(2.18) —or, more generally, the Hamiltonian (3.1) - in each subspace with well-defined spin content in terms of the supersymmetric version of Haldane's motifs [19] and their associated skew Young tableaux [20,23,41]. In particular, this description implies the validity of the Saiga-Kuramoto conjecture, which is one of the main results of this paper.

We start by recalling that the partition function (3.6) of the $\mathrm{su}(1 \mid \mathrm{m})$ spin chain (3.2) -and, hence, of the supersymmetric $\operatorname{su}(m) \mathrm{KY}$ model (3.1) - exactly coincides with the partition function of the inhomogeneous vertex model with energies $[22,23]$

$$
E(\mathbf{s})=J \sum_{i=1}^{N-1} \delta\left(s_{i}, s_{i+1}\right) i(N-i)-\sum_{i} \mu_{s_{i}}
$$

where $\mu_{0} \equiv 0, \mathbf{s} \in\{0, \ldots, m\}^{N}$ and $\delta\left(s, s^{\prime}\right)$ is defined by

$$
\delta\left(s, s^{\prime}\right)= \begin{cases}1, & s>s^{\prime} \text { or } s=s^{\prime}>0 \\ 0, & s<s^{\prime} \text { or } s=s^{\prime}=0\end{cases}
$$

The first sum in Eq. (4.1) can be interpreted as the energy of a one-dimensional vertex model with $N+1$ vertices $0, \ldots, N$ joined by $N$ bonds with values $s_{1}, \ldots, s_{N} \in$ $\{0, \ldots, m\}$, the energy associated to the $i$-th vertex being equal to $\delta\left(s_{i}, s_{i+1}\right) i(N-i)$. For this reason, we shall henceforth refer to the vector $\mathbf{s}$ as the bond vector. Likewise, the vectors $\boldsymbol{\delta}(\mathbf{s})$ with components $\delta\left(s_{i}, s_{i+1}\right)(1 \leqslant i \leqslant N-1)$ in Eq. (4.1) can be identified with $\mathrm{su}(1 \mid m)$ motifs $[19,23,27]$. Thus the spectrum of the $\mathrm{su}(m) \mathrm{KY}$ model (with the correct degeneracy for each level) can be computed from Eq. (4.1) by letting s run over all possible $(m+1)^{N}$ bond vectors. It is important to note that the energies (4.1) depend not only on the motif $\boldsymbol{\delta}$ but also on the chemical potentials $\mu_{\alpha}$ through the last term. This term will in general break the huge degeneracy associated to the motifs $\boldsymbol{\delta}$, 
which is in part due to the invariance of the model (3.3) with $\mu_{\alpha}=0$ (i.e., the $\mathrm{su}(1 \mid m)$ supersymmetric HS chain) under the Yangian $Y(\operatorname{gl}(1 \mid m))$ [41]. In other words, the general model (3.3) should be far less degenerate than the $\mathrm{su}(1 \mid m)$ supersymmetric HS spin chain.

Let us denote by $\psi_{\mathbf{s}}$ the unique eigenfunction of the supersymmetric $\operatorname{su}(m) \mathrm{KY}$ model (3.1) corresponding to the eigenvalue $E(\mathbf{s})$ (4.1) associated with the bond vector $\mathbf{s}$. Our aim is to determine the magnon numbers or spin content of the eigenfunction $\psi_{\mathbf{s}}$ directly from the structure of its bond vector s. To this end, we shall now show how the spectrum of the restriction of the Hamiltonian (3.1) to each subspace with well-defined spin content can be fully generated from the motif formula (4.1) by suitably restricting the components of the bond vector $\mathbf{s}$.

More precisely, let us denote by

$$
n\left(N_{0}, \ldots, N_{m}\right) \equiv n(\mathbf{N}), \quad N_{0}+\cdots+N_{m} \equiv|\mathbf{N}|=N,
$$

the subspace of the Hilbert space of the $\operatorname{su}(m)$ KY model in which each number operator $n^{\alpha}$ has a well-defined value $N_{\alpha}$. We shall henceforth refer to the vector $\mathbf{N}$ as the magnon content of the subspace $n(\mathbf{N})$. Note next that, by Eqs. (3.1) and (3.2), the KY Hamiltonian $H$ in Eq. (3.1) can be written as

$$
H=H_{0}^{\prime}-\sum_{\sigma} \mu_{\sigma} n^{\sigma}, \quad H_{0}^{\prime} \equiv H_{0}+t_{0} \sum_{\sigma} n^{\sigma},
$$

where $H_{0}$ and $t_{0}$ are respectively given by Eqs. (2.16)-(2.18) and (2.20). Clearly, $H_{0}^{\prime}$ does not depend on the chemical potentials $\mu_{\sigma}$ (since $H_{0}$ is also independent of $\mu_{\sigma}$ ); in fact, $\widehat{H_{0}^{\prime}}=J H_{\mathrm{HS}}^{(1 \mid m)}$. It should be noted at this point that the subspaces $n(\mathbf{N})$ are invariant under both $H$ and $H_{0}^{\prime}$, since the number operators $n^{\sigma}$ obviously commute with the operators $c_{k \sigma}^{\dagger} c_{l \sigma}, n_{k}, \mathbf{T}_{k} \cdot \mathbf{T}_{l}$. Consequently, by Eq. (4.2), the partition function of the Hamiltonian $H$ can be written as

$$
\mathcal{Z}(q ; \boldsymbol{\mu})=\sum_{\mathbf{N} ;|\mathbf{N}|=N} q^{-\sum_{\sigma} \mu_{\sigma} N_{\sigma}} \mathcal{Z}_{0}^{\mathbf{N}}(q),
$$

where $\mathcal{Z}_{0}^{\mathbf{N}}(q)$ denotes the partition function of the restriction of $H_{0}^{\prime}$ to the subspace $n(\mathbf{N})$. We also know that

$$
\mathcal{Z}(q ; \boldsymbol{\mu})=\mathcal{Z}_{V}(q ; \boldsymbol{\mu})
$$

where $\mathcal{Z}_{V}(q ; \boldsymbol{\mu})$ denotes the partition function of the vertex model (4.1). Let us now rewrite the latter equation as

$$
E(\mathbf{s})=J \sum_{i=1}^{N-1} \delta\left(s_{i}, s_{i+1}\right) i(N-i)-\sum_{\sigma} \mu_{\sigma} N_{\sigma}(\mathbf{s}),
$$

where $N_{\sigma}(\mathbf{s})$ denotes the number of components of the bond vector s equal to $\sigma$. We then have

$$
\mathcal{Z}_{V}(q ; \boldsymbol{\mu})=\sum_{\mathbf{s}} q^{E(\mathbf{s})}=\sum_{\mathbf{N} ;|\mathbf{N}|=N} q^{-\sum_{\sigma} \mu_{\sigma} N_{\sigma}} \sum_{\mathbf{s} ; N_{\alpha}(\mathbf{s})=N_{\alpha}} q^{J \sum_{i=1}^{N-1} \delta\left(s_{i}, s_{i+1}\right) i(N-i)},
$$

where the last sum is restricted to all bond vectors $\mathbf{s}$ such that $N_{\alpha}(\mathbf{s})=N_{\alpha}$ for all $\alpha=0, \ldots, m$. Note that the right-hand sides of Eqs. (4.3) and (4.5) are both 
polynomials in the variables $x_{\alpha} \equiv \mathrm{e}^{\beta \mu_{\alpha}}(\alpha=0, \ldots, m)$. Equating the coefficient of $x_{0}^{N_{0}} \cdots x_{m}^{N_{m}}$ in both equivalent expressions for $\mathcal{Z}(q ; \boldsymbol{\mu})$ we immediately deduce that the partition function of the restriction of $H_{0}^{\prime}$ to the subspace $n(\mathbf{N})$ is given by

$$
\mathcal{Z}_{0}^{\mathbf{N}}(q)=\sum_{\mathbf{s}: N_{\alpha}(\mathbf{s})=N_{\alpha}} q^{J \sum_{i=1}^{N-1} \delta\left(s_{i}, s_{i+1}\right) i(N-i)} .
$$

It follows from the latter equation that the spectrum of $H_{0}^{\prime}$ in the subspace $n(\mathbf{N})$ can be generated from the motif formula

$E_{0}(\mathbf{s} ; \mathbf{N})=J \sum_{i=1}^{N-1} \delta\left(s_{i}, s_{i+1}\right) i(N-i), \quad$ with $N_{\alpha}(\mathbf{s})=N_{\alpha}$ for all $\alpha=0, \ldots, m$.

In view of Eq. (4.2), the spectrum of the full Hamiltonian $H$ restricted to the subspace $n(\mathbf{N})$ (with the correct degeneracies for all levels) is generated by the analogous formula

$E(\mathbf{s} ; \mathbf{N})=E_{0}(\mathbf{s} ; \mathbf{N})-\sum_{\sigma} \mu_{\sigma} N_{\sigma}, \quad$ with $N_{\alpha}(\mathbf{s})=N_{\alpha}$ for all $\alpha=0, \ldots, m$.

We thus conclude that the spectrum of $H$ on $n(\mathbf{N})$ is obtained from Eq. (4.1) by imposing the natural conditions $N_{\alpha}(\mathbf{s})=N_{\alpha}$ on the bond vector $\mathbf{s}$. It follows from this assertion that we can label the eigenfunctions of $H$ in such a way that each $\psi_{\mathbf{s}}$ belongs to the subspace $n(\mathbf{N}(\mathbf{s}))$ containing exactly $N_{\alpha}(\mathbf{s})$ magnons of type $\alpha$. This is in fact one of the main results of this section, whose consequences we shall explore next.

To begin with, we shall use the method of Ref. [23] to express the spectrum (4.7) of the supersymmetric KY model (3.1) on the invariant subspace $n(\mathbf{N})$ in an alternative way. To this end, we first note that the numerical values of the energies can be computed from the formula

$$
E_{\boldsymbol{\delta}, \mathbf{N}}=J \sum_{i=1}^{N-1} \delta_{i} \cdot i(N-i)-\sum_{\sigma} \mu_{\sigma} N_{\sigma},
$$

where now $\boldsymbol{\delta} \equiv\left(\delta_{1}, \ldots, \delta_{N-1}\right)$ is a supersymmetric motif, i.e., a sequence of $N-1$ zeros and ones. Secondly, the degeneracy of each energy $E_{\boldsymbol{\delta}, \mathbf{N}}$ (which could be zero) is evaluated by counting the number of ways of filling the border strip associated to the motif $\boldsymbol{\delta}$ according to the usual $\mathrm{su}(1 \mid m)$ rules [41,42], with the additional restriction that each number $\alpha \in\{0, \ldots, m\}$ must appear exactly $N_{\alpha}$ times. More precisely, given the motif $\left(\delta_{1}, \ldots, \delta_{N-1}\right)$ its associated border strip is constructed by starting with one box, and then reading the motif from left to right and adding a box below (resp. to the left of) the $i$-th box provided that $\delta_{i}$ is equal to 0 (resp. 1); see, e.g., Fig. 1. This border strip should then be filled with the numbers $0,1, \ldots, m$ according to the following rules:

i) The numbers in each row form a nondecreasing sequence, allowing only the repetition of positive numbers.

ii) The numbers in each column (read from top to bottom) form a nondecreasing sequence, allowing only the repetition of 0 .

iii) Each number $\alpha \in\{0, \ldots, m\}$ must appear exactly $N_{\alpha}$ times. 

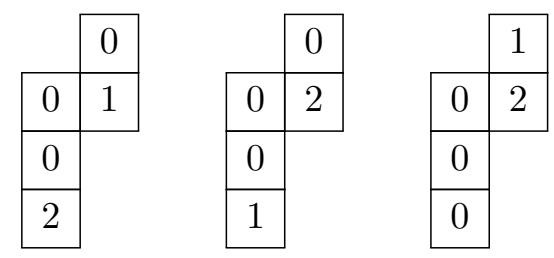

Figure 1. Allowed Young tableaux for the motif $(0,1,0,0)$ in the subspace $n(3,1,1)$.

Each filling of a border strip according to the previous rules is called a (skew) Young tableau. Given such a tableau, it is straightforward to check that its associated motif can be obtained from the bond vector $\left(s_{1}, \ldots, s_{N}\right)$ whose components are the numbers in the tableau read from top to bottom by setting $\delta_{i}=\delta\left(s_{i}, s_{i+1}\right)$. The equivalence between the description of the spectrum through Eq. (4.8) (where the degeneracy of a motif $\boldsymbol{\delta}$ is evaluated counting all the fillings of its associated border strip allowed by rules i)-iii)) and Eq. (4.7) essentially follows from this observation. Note also that, by Eq. (4.8), all the Young tableaux associated to a given motif have the same energy within each invariant subspace $n(\mathbf{N})$.

Consider, for instance, the motif $\boldsymbol{\delta}=(0,1,0,0)$ for $N=5$ particles in the $\operatorname{su}(1 \mid 2)$ case. As explained above, the degeneracy associated to this motif in each invariant subspace $n(\mathbf{N})$ is given by all possible Young tableaux generated from it according to the above three rules. For instance, for the subspace $n(3,1,1)$ it is easy to check that there are exactly three allowed Young tableaux for the above motif (cf. Fig. 1), with energy $6 J-\mu_{1}-\mu_{2}$. In fact, it is a straightforward matter to verify that there are exactly 8 invariant subspaces $n(\mathbf{N})$ with fixed spin content compatible with the motif $(0,1,0,0)$ (i.e., with nonzero degeneracy), whose respective degeneracies and energies are listed in Table 1.

\begin{tabular}{|c|c|c|}
\hline $\mathbf{N}$ & Degeneracy & Energy \\
\hline$(1,2,2)$ & 1 & $6 J-2\left(\mu_{1}+\mu_{2}\right)$ \\
$(3,0,2)$ & 1 & $6 J-2 \mu_{2}$ \\
$(3,2,0)$ & 1 & $6 J-2 \mu_{1}$ \\
$(4,0,1)$ & 1 & $6 J-\mu_{2}$ \\
$(4,1,0)$ & 1 & $6 J-\mu_{1}$ \\
$(2,1,2)$ & 2 & $6 J-\left(\mu_{1}+2 \mu_{2}\right)$ \\
$(2,2,1)$ & 2 & $6 J-\left(2 \mu_{1}+\mu_{2}\right)$ \\
$(3,1,1)$ & 3 & $6 J-\left(\mu_{1}+\mu_{2}\right)$ \\
\hline
\end{tabular}

Table 1. Invariant subspaces $n(\mathbf{N})$ compatible with the motif $(0,1,0,0)$ in the $\operatorname{su}(1 \mid 2)$ case.

The above general results considerably simplify for the supersymmetric $\operatorname{su}(m) \mathrm{KY}$ model without magnetic field or chemical potential terms, given by Eq. (2.16)-(2.18). 


\begin{tabular}{|c|c|c|c|c|c|c|c|}
\hline Motif & 0 & 1 & 2 & 3 & 4 & 5 & Energy \\
\hline$(0,0,0,0)$ & 0 & 0 & 0 & 1 & 2 & 1 & $-4 J\left(5-N_{0}\right)$ \\
$(0,0,0,1),(1,0,0,0)$ & 0 & 0 & 2 & 4 & 2 & 0 & $-4 J\left(4-N_{0}\right)$ \\
$(0,0,1,0),(0,1,0,0)$ & 0 & 1 & 4 & 5 & 2 & 0 & $-2 J\left(7-2 N_{0}\right)$ \\
$(1,0,0,1)$ & 0 & 4 & 8 & 4 & 0 & 0 & $-4 J\left(3-N_{0}\right)$ \\
$(0,0,1,1),(1,1,0,0)$ & 0 & 3 & 6 & 3 & 0 & 0 & $-2 J\left(5-2 N_{0}\right)$ \\
$(1,0,1,0),(0,1,0,1)$ & 2 & 8 & 10 & 4 & 0 & 0 & $-2 J\left(5-2 N_{0}\right)$ \\
$(0,1,1,0)$ & 2 & 7 & 8 & 3 & 0 & 0 & $-4 J\left(2-N_{0}\right)$ \\
$(1,0,1,1),(1,1,0,1)$ & 6 & 12 & 6 & 0 & 0 & 0 & $-2 J\left(3-2 N_{0}\right)$ \\
$(0,1,1,1),(1,1,1,0)$ & 4 & 8 & 4 & 0 & 0 & 0 & $-4 J\left(1-N_{0}\right)$ \\
$(1,1,1,1)$ & 6 & 5 & 0 & 0 & 0 & 0 & $4 J N_{0}$ \\
\hline
\end{tabular}

Table 2. Spectrum of the $\mathrm{su}(1 \mid 2) \mathrm{KY}$ model (2.16)-(2.18) with $N=5$ sites. The integers appearing in the columns labeled $0, \ldots, 5$ are the degeneracies of the motif(s) in each row for the subspaces with $N_{0}=0, \ldots, 5$ holes.

Indeed, in this case $h_{\sigma}=\mu_{c}=0$, so that from Eq. (3.5) we obtain

$$
\mu_{\sigma}=t_{0}=\frac{t \pi^{2}}{3 N^{2}}\left(N^{2}-1\right), \quad 1 \leqslant \sigma \leqslant m .
$$

Thus Eq. (4.8) becomes

$$
E_{\boldsymbol{\delta}, \mathbf{N}}=J \sum_{i=1}^{N-1} \delta_{i} \cdot i(N-i)-t_{0}\left(N-N_{0}\right)=J\left[\sum_{i=1}^{N-1} \delta_{i} \cdot i(N-i)-\frac{1}{6}\left(N^{2}-1\right)\left(N-N_{0}\right)\right],
$$

which depends on the spin content $\mathbf{N}$ only through $N_{0}$. Thus for a given motif $\boldsymbol{\delta}$ all of its compatible invariant subspaces $n(\mathbf{N})$ with the same number of holes $N_{0}$ will now have the same energy. For instance, for the case $N=5, m=2$ and the motif $(0,1,0,0)$ considered above there are only four sectors with different energies, corresponding to $N_{0}=1$ (singlet), 2 (four times degenerate), 3 (five times degenerate) and 4 (twice degenerate), with respective energies $-2 J\left(7-2 N_{0}\right)$. In general, in order to compute the degeneracy associated to each motif $\boldsymbol{\delta}$ in a sector with a given number of holes $N_{0}$ we just have to count the number of allowed Young tableaux according to rules i) and ii) above, replacing rule three by the following:

iii') The number 0 must appear exactly $N_{0}$ times.

The rules i), ii), iii') provide a complete description of the spectrum of the supersymmetric $\mathrm{su}(m) \mathrm{KY}$ model (2.16)-(2.18), with the correct degeneracy for each energy (4.9), for arbitrary values of $m$ and the number of sites $N$. In particular, when applied to the simplest $\mathrm{su}(2)$ case these rules provide the first rigorous proof known to the authors to the long-standing conjecture of Saiga and Kuramoto mentioned in the Introduction.

As a simple illustration of the above assertion, we present in Table 2 the detailed spectrum of the (original) $\mathrm{su}(2) \mathrm{KY}$ model (2.16)-(2.18) with $N=5$ sites. More precisely, in each of the columns of this table labeled with the integers $N_{0}=0, \ldots, 5$ 
we list the degeneracy associated to the motif(s) in each row for the subspace with $N_{0}$ holes. This degeneracy is computed by first generating all the skew Young tableaux compatible with each of the $2^{N-1}=16$ possible motifs according to rules i), ii) and iii') above, which can be easily accomplished using a simple Mathematica program. We then sort the resulting tableaux according to the number of holes (zeros) in each of them. By Eq. (4.9), a motif $\boldsymbol{\delta}=\left(\delta_{1}, \ldots, \delta_{N-1}\right)$ and its reverse $\boldsymbol{\delta}^{\prime}=\left(\delta_{N-1}, \ldots, \delta_{1}\right)$ clearly have the same energy. In fact, it can be shown that two such motifs give rise to the same numbers of compatible Young tableaux in each subspace $n(\mathbf{N})$ (see the appendix for details). For this reason, we have grouped together in Table 2 two motifs that are the reverse of each other. More generally, if we exchange any two components $\delta_{k}$ and $\delta_{N-k}$ of a motif $\boldsymbol{\delta}$ we obtain a motif with the same energy as $\boldsymbol{\delta}$ in each subspace with $N_{0}$ holes. However, these two motifs may not necessarily yield the same number of compatible Young tableaux in such a subspace. For instance, the motifs $(0,0,1,1)$ and $(1,0,1,0)$ have the same energy $-2 J\left(5-2 N_{0}\right)$ in a subspace with $N_{0}$ holes, but it is clear from Table 2 that their degeneracies differ in each of these subspaces for $N_{0}=0, \ldots, 3$. Finally, it is apparent from Table 2 that the ground state is obtained from the motifs $(0,0,1,0),(0,1,0,0)$ (in the sector with one hole) and $(1,0,1,0),(0,1,0,1)$ (in the sector with no holes). It is thus six times degenerate, with energy $-10 \mathrm{~J}$.

\section{Ground state phases for the supersymmetric spin 1/2 KY model}

The complete description of the spectrum of the supersymmetric KY model in terms of motifs, bond vectors and their associated skew Young tableaux developed in the previous section is particularly suited to studying its ground state. As an example, we shall compute next the ground state energy per site of the spin $1 / 2 \mathrm{KY}$ model in the thermodynamic limit for all possible values of the magnetic field strength $h \equiv h_{1}$ and chemical potential $\mu \equiv \mu_{c}+t_{0}$. To this end, let us first choose the unit of energy so that $t=1 /\left(2 \pi^{2}\right)$, so that Eq. (4.8) reads

$$
E_{\boldsymbol{\delta}, \mathbf{N}}=\frac{1}{N^{2}} \sum_{i=1}^{N-1} \delta_{i} \cdot i(N-i)-\frac{h}{2}\left(N_{1}-N_{2}\right)-\mu\left(N_{1}+N_{2}\right) .
$$

We shall also assume without loss of generality that $h \geqslant 0$, since taking $h<0$ simply reverses the role of the "up" $(\sigma=1)$ and "down" $(\sigma=2)$ fermions. It is then clear from the term proportional to $h$ in Eq. (5.1) that the ground state(s) must belong to an invariant subspace $n(\mathbf{N})$ with $N_{1} \geqslant N_{2}$. Since the dispersion function $i(N-i)$ is symmetric about $i=N / 2$ and has an absolute maximum at this point, by Eq. (4.1) for $N$ large enough the bond vector minimizing the energy in the subspace $n(\mathbf{N})$ must be 
of the form $\S$

$$
\mathbf{s}_{0}=(\underbrace{1 \cdots 1}_{\left(N_{1}-N_{2}\right) / 2} \underbrace{12 \cdots 12}_{N_{2}} \underbrace{0 \cdots 0}_{N_{0}} \underbrace{12 \cdots 12}_{N_{2}} \underbrace{1 \cdots 1}_{\left(N_{1}-N_{2}\right) / 2}) .
$$

By Eq. (5.1), the energy per site of the corresponding motif

$$
\boldsymbol{\delta}_{0}=(\underbrace{1, \ldots, 1}_{\left(N_{1}-N_{2}\right) / 2} \underbrace{0,1, \ldots, 0,1}_{N_{2}} \underbrace{0, \ldots, 0}_{N_{0}} \underbrace{0,1, \ldots, 0,1}_{N_{2}} \underbrace{1, \ldots, 1}_{\left(N_{1}-N_{2}\right) / 2-1})
$$

is given by

$$
\frac{E_{\boldsymbol{\delta}_{0}, \mathbf{N}}}{N}=\frac{2}{N} \sum_{k=1}^{N t} \varepsilon\left(x_{k}\right)+\frac{2}{N} \sum_{k=1}^{N(s-t) / 2} \varepsilon\left(x_{N t+2 k}\right)-h t-2 \mu s \equiv u_{\boldsymbol{\delta}_{0}, \mathbf{N}},
$$

where the factor of 2 before the sums comes from the obvious symmetry of $\boldsymbol{\delta}_{0}$ around $N / 2$ and we have set

$$
s=\frac{N_{1}+N_{2}}{2 N}, \quad t=\frac{N_{1}-N_{2}}{2 N}, \quad x_{k}=\frac{k}{N}, \quad \varepsilon(x)=x(1-x) .
$$

Hence in the thermodynamic limit the minimum energy per site in a subspace $n(\mathbf{N})$ with $N_{1} \geqslant N_{2}$ is given by

$$
\begin{aligned}
u(s, t) \equiv \lim _{N \rightarrow \infty} u_{\boldsymbol{\delta}_{0}, \mathbf{N}} & =2 \int_{0}^{t} \varepsilon(x) \mathrm{d} x+\int_{t}^{s} \varepsilon(x) \mathrm{d} x-h t-2 \mu s \\
& =\int_{0}^{s} \varepsilon(x) \mathrm{d} x+\int_{0}^{t} \varepsilon(x) \mathrm{d} x-h t-2 \mu s \equiv f_{2 \mu}(s)+f_{h}(t),
\end{aligned}
$$

where

$$
f_{\lambda}(s)=\int_{0}^{s} \varepsilon(x) \mathrm{d} x-\lambda s \equiv f(s)-\lambda s .
$$

The ground state energy of the spin $1 / 2 \mathrm{KY}$ model in the thermodynamic limit is thus the minimum value of the function $u(s, t)$ in the triangle

$$
D=\left\{(s, t) \in \mathbb{R}^{2}: 0 \leqslant t \leqslant s \leqslant 1 / 2\right\} .
$$

In order to compute this minimum value, note first of all that $f_{\lambda}^{\prime}(x)=\varepsilon(x)-\lambda$, with $\varepsilon(x)$ monotonically increasing from 0 to $1 / 4$ in the interval $[0,1 / 2]$. Hence $f_{\lambda}$ is monotonically increasing $\|$ over the interval $[0,1 / 2]$ for $\lambda \leqslant 0$, monotonically decreasing for $\lambda \geqslant 1 / 4$, and has a unique global minimum at the point

$$
x_{0}(\lambda) \equiv \varepsilon^{-1}(\lambda)=\frac{1}{2}(1-\sqrt{1-4 \lambda}) \in(0,1 / 2)
$$

for $0<\lambda<1 / 4$. We thus have the following possibilities (recall that we are assuming throughout that $h \geqslant 0)$ :

$\S$ We are actually assuming here that both $N_{1}$ and $N_{2}$ are even. In other cases the form of the minimizing bond vector differs slightly from Eq. (5.2), but the formula for its energy coincides in the thermodynamic limit with the one given below. Note also that unless $N_{1}$ and $N_{2}$ are both odd there is actually an additional bond vector with the same energy as (5.2) (or its variants, for $N_{1}$ and $N_{2}$ of opposite parity).

$\|$ For the sake of conciseness, we shall implicitly assume in what follows that the functions $f_{\lambda}$ and $\varepsilon$ are restricted to the interval of interest $[0,1 / 2]$. 
i) $\frac{h}{2}<\mu<1 / 8$

In this case $f_{h}(t)$ and $f_{2 \mu}(s)$ both have a unique minimum over the interval $[0,1 / 2]$ respectively at the points $t_{0}=x_{0}(h) \in[0,1 / 2)$ and $s_{0}=x_{0}(2 \mu) \in(0,1 / 2)$, with $t_{0}<s_{0}$ since $\varepsilon\left(t_{0}\right)=h<2 \mu=\varepsilon\left(s_{0}\right)$. Hence $u$ attains its global minimum on $D$ at the point $\left(s_{0}, t_{0}\right)$ (which is an interior point if $h>0$ ), and the ground state energy density $u$ is consequently given by

$$
\begin{aligned}
u & =u\left(s_{0}, t_{0}\right)=\int_{0}^{x_{0}(h)} \varepsilon(x) \mathrm{d} x+\int_{0}^{x_{0}(2 \mu)} \varepsilon(x) \mathrm{d} x-h x_{0}(h)-2 \mu x_{0}(2 \mu) \\
& =\frac{1}{6}\left[1-3(h+2 \mu)-\frac{1}{2}(1-4 h)^{3 / 2}-\frac{1}{2}(1-8 \mu)^{3 / 2}\right] .
\end{aligned}
$$

Note also that $t=\left(N_{1}-N_{2}\right) /(2 N)$ and $2 s=\left(N_{1}+N_{2}\right) / N$ are respectively equal to the magnetization and the charge density per site (assuming that the fermions have unit charge and gyromagnetic ratio equal to 2). Hence the zero-temperature magnetization and charge densities are given by

$$
m_{s}=x_{0}(h)=\frac{1}{2}(1-\sqrt{1-4 h}), \quad n_{c}=2 x_{0}(2 \mu)=1-\sqrt{1-8 \mu} .
$$

The corresponding magnetic and charge susceptibilities are obtained by differentiation, namely,

$\chi_{s}=\frac{\partial m_{s}}{\partial h}=(1-4 h)^{-1 / 2}=\left(1-2 m_{s}\right)^{-1}, \quad \chi_{c}=\frac{\partial n_{c}}{\partial \mu}=4(1-8 \mu)^{-1 / 2}=4\left(1-n_{c}\right)^{-1}$,

in agreement with known results (see, e.g., [25]).

If $(h, \mu)$ lies outside the region $\left\{(h, \mu) \in \mathbb{R}^{2}: 0 \leqslant h / 2<\mu<1 / 8\right\}$, the system

$$
\varepsilon(s)=2 \mu, \quad \varepsilon(t)=h
$$

determining the critical points of $u$ has no solutions within the interior of $D$. Thus the function $u(s, t)$ must necessarily attain its minimum value in the triangle $D$ on its sides. It is worth noting in this respect that this minimum cannot be reached at an interior point of the horizontal side $t=0$ unless $h=0$, since for $h>0$ we have $f_{h}^{\prime}(0)=-h<0$ and thus

$$
u(s, 0)=f_{2 \mu}(s)>f_{2 \mu}(s)+f_{h}(t)=u(s, t)
$$

for sufficiently small $t>0$. We are left with the following possibilities:

ii) $h \geqslant 1 / 4, \mu+\frac{h}{2} \geqslant 1 / 4$

In this case $u(s, s)=f_{h}(s)+f_{2 \mu}(s)=2 f_{h / 2+\mu}(s)$ and $u(1 / 2, t)=f_{h}(t)+f_{2 \mu}(1 / 2)$ are both decreasing, so that these functions have a unique global minimum on the interval $[0,1 / 2]$ at the right endpoint $1 / 2$, with common value

$$
u(1 / 2,1 / 2)=2 f_{h / 2+\mu}(1 / 2)=\frac{1}{6}[1-3(h+2 \mu)] .
$$

This must be the unique minimum of $u$ on $D$, since as $h>0$ this minimum cannot be attained on the interior of the side $t=0$. Hence in this case the ground state energy per 
site is given by Eq. (5.5), while the zero-temperature magnetization and charge densities are simply

$$
n_{c}=2 m_{s}=1 \text {. }
$$

Note that $s=t=1 / 2$ is equivalent to $N_{1}=N$, so that this phase consists only of up fermions.

iii) $\mu+\frac{h}{2} \leqslant 0$

Since $\mu \leqslant-h / 2 \leqslant 0$, the minimum of $u(s, 0)=f_{2 \mu}(s)$ and $u(s, s)=2 f_{h / 2+\mu}(s)$ on $[0,1 / 2]$ is located at $s=0$, while

$$
u(1 / 2, t)=f(t)+f(1 / 2)-h t-\mu>-\frac{h}{2}-\mu \geqslant 0=u(0,0) .
$$

Thus in this case the ground state energy, zero-temperature magnetization and charge all vanish. In fact, since $s=t=0$ is equivalent to $N_{0}=N$, this is the trivial phase consisting only of holes.

iv) $h<1 / 4, \mu \geqslant 1 / 8$

In this case $u(1 / 2, t)$ attains its minimum value in the interval $[0,1 / 2]$ at $t_{0}=x_{0}(h) \in$ $[0,1 / 2)$. On the other hand,

$$
u(s, s)=f_{h}(s)+f_{2 \mu}(s)>f_{h}\left(t_{0}\right)+f_{2 \mu}(1 / 2)=u\left(1 / 2, t_{0}\right),
$$

since $f_{2 \mu}$ is decreasing in the interval $[0,1 / 2]$ on account of the condition $\mu \geqslant 1 / 8$. Similarly,

$$
u(s, 0)=f_{2 \mu}(s) \geqslant f_{2 \mu}(1 / 2)=u(1 / 2,0) \geqslant u\left(1 / 2, t_{0}\right),
$$

with equality only if $h=0$ and $s=1 / 2$. Thus the global minimum of $u$ on $D$ is attained at the point $\left(1 / 2, t_{0}\right)$. In particular, the ground state energy per site is given by

$$
\begin{aligned}
u=u\left(1 / 2, t_{0}\right) & =\int_{0}^{x_{0}(h)} \varepsilon(x) \mathrm{d} x-h x_{0}(h)+\int_{0}^{1 / 2} \varepsilon(x) \mathrm{d} x-\mu \\
& =\frac{1}{6}\left[1-3(h+2 \mu)-\frac{1}{2}(1-4 h)^{3 / 2}\right],
\end{aligned}
$$

while the magnetization and charge per site at zero temperature read

$$
m_{s}=x_{0}(h), \quad n_{c}=1 .
$$

The $T=0$ magnetic susceptibility is again

$$
\chi_{s}=(1-4 h)^{-1 / 2}=\left(1-2 m_{s}\right)^{-1},
$$

while the charge susceptibility vanishes. Since $s=1 / 2$ is equivalent to $N_{1}+N_{2}=N$, this is an $\mathrm{su}(2)$ phase characterized by the absence of holes.

v) $0<h / 2+\mu<1 / 4, \mu \leqslant h / 2$

The above inequalities imply that $u(s, s)=2 f_{h / 2+\mu}(s)$ attains its unique global minimum over the interval $[0,1 / 2]$ at the point $s_{0}=x_{0}(h / 2+\mu) \in(0,1 / 2)$. It is straightforward to check that $u(s, t)$ achieves its minimum over the domain $D$ at the point $\left(s_{0}, s_{0}\right)$. Indeed, 


\begin{tabular}{|c|c|c|}
\hline Region & Ground state energy per site & Spin content \\
\hline$\frac{h}{2}<\mu<\frac{1}{8}$ & $\frac{1}{6}\left[1-3(h+2 \mu)-\frac{1}{2}(1-4 h)^{3 / 2}-\frac{1}{2}(1-8 \mu)^{3 / 2}\right]$ & $B, F_{1}, F_{2}$ \\
\hline$h \geqslant \frac{1}{4}, \quad \mu+\frac{h}{2} \geqslant \frac{1}{4}$ & $\frac{1}{6}[1-3(h+2 \mu)]$ & $F_{1}$ \\
\hline$\mu+\frac{h}{2} \leqslant 0$ & 0 & $B$ \\
\hline$h<\frac{1}{4}, \quad \mu \geqslant \frac{1}{8}$ & $\frac{1}{6}\left[1-3(h+2 \mu)-\frac{1}{2}(1-4 h)^{3 / 2}\right]$ & $F_{1}, F_{2}$ \\
\hline $0<\mu+\frac{h}{2}<\frac{1}{4}, \quad \mu \leqslant \frac{h}{2}$ & $\frac{1}{6}\left[1-3(h+2 \mu)-(1-2 h-4 \mu)^{3 / 2}\right]$ & $B, F_{1}$ \\
\hline
\end{tabular}

Table 3. Ground state energy per site and spin content $\left(B \equiv\right.$ hole (boson), $F_{\sigma} \equiv$ fermion of type $\sigma$ ) for the spin $1 / 2 \mathrm{KY}$ model as a function of its parameters $h \geqslant 0$ and $\mu$ (the unit of energy has been taken as $2 \pi^{2} t$ ).

as $h>0$ in this case this minimum cannot be reached on the interior of the side $t=0$. Consider next the side $s=1 / 2$. If $h \geqslant 1 / 4$ the function $f_{h}(t)$ is decreasing, so that $u(1 / 2, t)=f_{h}(t)+f_{2 \mu}(1 / 2) \geqslant f_{h}(1 / 2)+f_{2 \mu}(1 / 2)=u(1 / 2,1 / 2)>u\left(s_{0}, s_{0}\right)$.

On the other hand, if $0 \leqslant h<1 / 4$ then $f_{h}$ has a global minimum on $[0,1 / 2]$ at $t_{0}=x_{0}(h) \geqslant x_{0}(h / 2+\mu)=s_{0}$, with $t_{0} \in[0,1 / 2)$. Since $\varepsilon(x)-2 \mu>0$ for $x>t_{0}$ (this is obvious for $\mu \leqslant 0$, while for $0<\mu \leqslant 1 / 8$ it is a consequence of the inequality $x_{0}(2 \mu) \leqslant x_{0}(h)$, which in turn follows from $\left.2 \mu \leqslant h\right)$ we have

$$
f_{2 \mu}(1 / 2)=\int_{0}^{1 / 2}(\varepsilon(x)-2 \mu) \mathrm{d} x>\int_{0}^{t_{0}}(\varepsilon(x)-2 \mu) \mathrm{d} x=f_{2 \mu}\left(t_{0}\right)
$$

Hence

$$
\begin{aligned}
u\left(s_{0}, s_{0}\right)-u\left(1 / 2, t_{0}\right) & =2 f_{h / 2+\mu}\left(s_{0}\right)-f_{h}\left(t_{0}\right)-f_{2 \mu}(1 / 2) \\
& <2 f_{h / 2+\mu}\left(s_{0}\right)-f_{h}\left(t_{0}\right)-f_{2 \mu}\left(t_{0}\right)=2 f_{h / 2+\mu}\left(s_{0}\right)-2 f_{h / 2+\mu}\left(t_{0}\right) \leqslant 0,
\end{aligned}
$$

which completes the proof of our assertion. In summary, in this case the ground state energy per site is given by

$$
\begin{aligned}
u=u\left(s_{0}, s_{0}\right) & =2 \int_{0}^{x_{0}(h / 2+\mu)} \varepsilon(x) \mathrm{d} x-(h+2 \mu) x_{0}\left(\frac{h}{2}+\mu\right) \\
& =\frac{1}{6}\left[1-3(h+2 \mu)-(1-2 h-4 \mu)^{3 / 2}\right]
\end{aligned}
$$

while the magnetization, the charge density and their susceptibilities (per site) read

$$
\begin{aligned}
& n_{c}=2 m_{s}=2 x_{0}(h / 2+\mu)=1-\sqrt{1-2 h-4 \mu}, \\
& \chi_{c}=4 \chi_{s}=2(1-2 h-4 \mu)^{-1 / 2}=\frac{2}{1-n_{c}}=\frac{2}{1-2 m_{s}} .
\end{aligned}
$$

Note, finally, that the equality $s=t$ is equivalent to $N_{2}=0$. This is thus an $\operatorname{su}(1 \mid 1)$ phase, consisting only of holes and up fermions.

Our results are summarized in Table 3. Note, in particular, that $u$ is continuous (indeed, of class $C^{1}$ ) over its domain, although its second derivatives are discontinuous along the boundaries of the regions listed in Table 3 (cf. Fig. 2). 

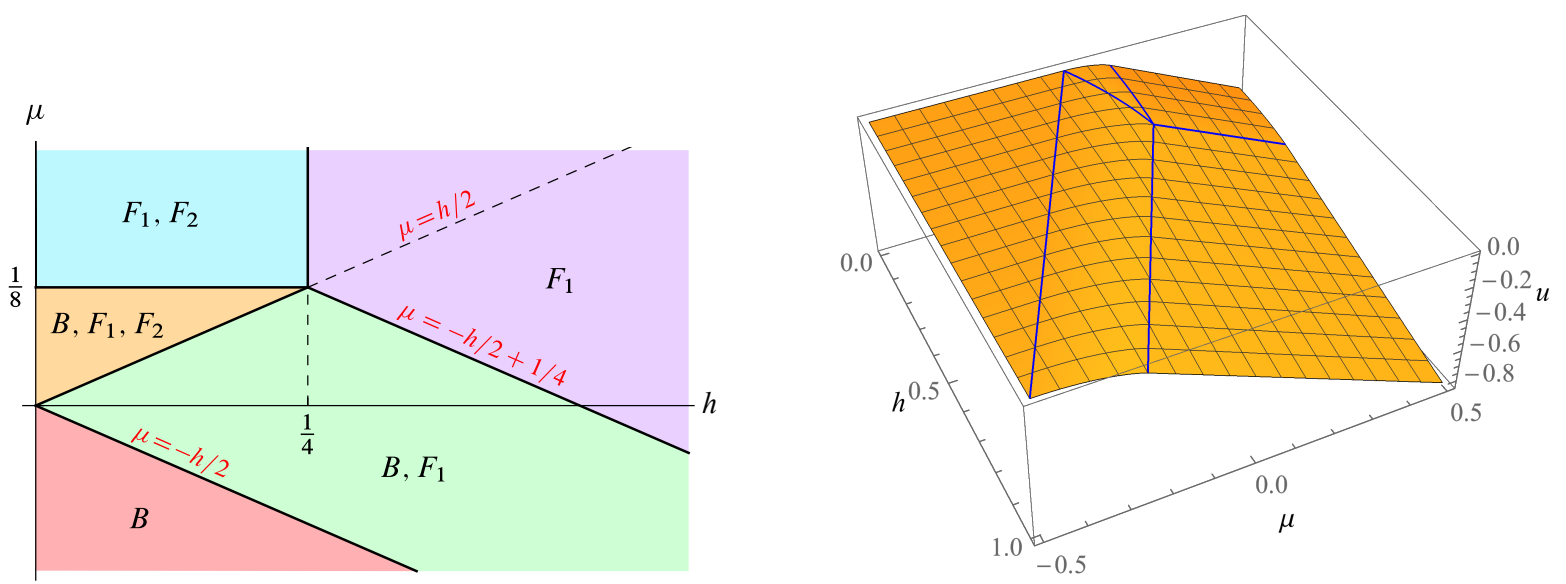

Figure 2. Left: phase diagram of the ground state of the spin $1 / 2 \mathrm{KY}$ model $(B \equiv$ hole (boson), $F_{\sigma} \equiv$ fermion of type $\sigma$ ). Right: ground state energy per site of the latter model as a function of its parameters $h \geqslant 0$ and $\mu$ (the blue lines represent the boundaries of the regions in the phase diagram). In both plots, the unit of energy has been taken as $2 \pi^{2} t$.

\section{Conclusions}

Although the supersymmetric $\mathrm{su}(m) t-J$ model with long-range interactions, also known as the $\mathrm{su}(m) \mathrm{KY}$ model, has been studied extensively during the last few decades, an analytical derivation of its spectrum and exact partition function has been missing so far. With the purpose of filling up this gap, in this paper we first establish the precise equivalence of the $\mathrm{su}(m) \mathrm{KY}$ model to a suitable modification of the $\mathrm{su}(1 \mid \mathrm{m})$ HS spin chain with chemical potential terms. This equivalence allows us to obtain the partition function of the former model from that of the latter, which was recently computed in Ref. [22]. A remarkable property of this partition function is the fact that it can be rewritten as the partition function of a suitable inhomogeneous vertex model. Analyzing the structure of these two equivalent partition functions, we not only obtain the complete spectrum of the $\mathrm{su}(m) \mathrm{KY}$ model in the presence of an arbitrary magnetic field and charge chemical potential, but also develop a novel method for determining the magnon numbers or spin content of the corresponding wave functions. This yields an exhaustive description of the spectrum in the subspaces with well-defined magnon content in terms of suitably restricted bond configurations of the equivalent vertex model, which are closely connected with supersymmetric versions of Haldane's motifs and their related skew Young tableaux. For the particular case $m=2$, this description provides a rigorous proof of a long-standing conjecture by Saiga and Kuramoto [21] based on numerical evidence.

As a concrete application of our results, we study various thermodynamic properties of the su(2) KY model in the zero temperature limit. To this end, we determine the structure of the motifs and bond configurations yielding the ground state of the latter model in the thermodynamic limit for different values of the external parameters. The structure of such bond configurations leads to a complete description of the different 
ground state phases in terms of the magnetic field strength and the charge chemical potential. These phases are characterized by the spin content (magnon numbers) of the corresponding wave functions, namely an $\mathrm{su}(1 \mid 2)$ phase where holes and fermions of both types co-exist, an $\mathrm{su}(1 \mid 1)$ phase with holes and fermions of only one type, and an $\mathrm{su}(0 \mid 2)$ phase with fermions of both types, apart from the trivial phases consisting of only holes or fermions of one type. We also compute the zero-temperature values of the energy, magnetization and charge density, along with the magnetic and charge susceptibilities, for each ground state phase. This description of the thermodynamic properties at zero temperature goes beyond previously known results, which were derived by different methods and restricted to the $\mathrm{su}(1 \mid 2)$ phase. In particular, our analysis confirms that the strong spin-charge separation characteristic of the long-range $t$ - $J$ model at low temperatures [25] occurs in all nontrivial phases.

Note, finally, that the description of the spectrum of the su $(m)$ KY model in terms of supersymmetric motifs and their associated Young tableaux derived in this paper makes it possible to compute in closed form the model's thermodynamic functions at finite temperature, by means of the transfer matrix method developed in Refs. [22,43]. In fact, work on this problem is currently in progress and shall be presented in a forthcoming publication.

\section{Acknowledgments}

We would like to thank the anonymous referee, whose remarks helped to improve the presentation. This work was partially supported by Spain's MINECO grant FIS201563966-P. JAC would also like to acknowledge the financial support of the Universidad Complutense de Madrid through a 2015 predoctoral scholarship.

\section{Appendix A. Degeneracy of a reverse motif}

In this appendix we shall show that the degeneracy $d(\boldsymbol{\delta}, \mathbf{N})$ of the motif $\boldsymbol{\delta}=$ $\left(\delta_{1}, \ldots, \delta_{N-1}\right)$ in the subspace $n(\mathbf{N})$, i.e., the number of allowed Young tableaux for this motif containing $N_{\alpha}$ instances of each of the integer $\alpha \in\{0, \ldots, m\}$, is equal to that of its reverse $\boldsymbol{\delta}^{\prime} \equiv\left(\delta_{N-1}, \ldots, \delta_{1}\right)$. To this end, define the super-Schur polynomial associated to the motif $\boldsymbol{\delta}$ by

$$
S_{\boldsymbol{\delta}}(x, \mathbf{y})=\sum_{T \in \boldsymbol{\delta}} x^{N_{0}(T)} y_{1}^{N_{1}(T)} \cdots y_{m}^{N_{m}(T)},
$$

where the sum is extended to all allowed Young tableaux $T$ associated to $\boldsymbol{\delta}$ according to rules i)-iii) in Section 4 , and $N_{\alpha}(T)$ denotes the number of times the integer $\alpha$ appears in $T$. It is well known (see, e.g., $[20,27,42]$ ) that this polynomial can be computed from 
the determinantal formula

$$
S_{\delta}=\left|\begin{array}{ccccc}
E_{k_{r}} & E_{k_{r-1}+k_{r}} & E_{k_{r-2}+k_{r-1}+k_{r}} & \cdots & E_{k_{1}+\cdots+k_{r}} \\
1 & E_{k_{r-1}} & E_{k_{r-2}+k_{r-1}} & \cdots & E_{k_{1}+\cdots+k_{r-1}} \\
0 & 1 & E_{k_{r-2}} & \cdots & E_{k_{1}+\cdots+k_{r-2}} \\
\vdots & \vdots & \vdots & \vdots & \vdots \\
0 & \cdots & 1 & E_{k_{2}} & E_{k_{1}+k_{2}} \\
0 & \cdots & 0 & 1 & E_{k_{1}}
\end{array}\right| \equiv S\left\langle k_{1}, \ldots, k_{r}\right\rangle,
$$

where $\sum_{j=1}^{i} k_{j}$ (with $1 \leqslant i \leqslant r-1$ ) denotes the position of the $i$-th 1 in the motif $\boldsymbol{\delta}$,

$$
k_{r}=N-\sum_{i=1}^{r-1} k_{i},
$$

and the polynomials $E_{k}(x, \mathbf{y})$ are defined in terms of the elementary symmetric polynomials (3.8) by

$$
E_{k}(x, \mathbf{y})=\sum_{l=0}^{k} x^{k-l} e_{l}(\mathbf{y}) .
$$

Clearly, for the reverse motif $\boldsymbol{\delta}^{\prime}$ we have $k_{i}^{\prime}=k_{r+1-i}$, so that

$$
S_{\boldsymbol{\delta}^{\prime}}=S\left\langle k_{r}, \ldots, k_{1}\right\rangle .
$$

Since, by definition of $S_{\boldsymbol{\delta}}, d(\boldsymbol{\delta}, \mathbf{N})$ is the coefficient of $x^{N_{0}} y_{1}^{N_{1}} \cdots y_{m}^{N_{m}}$ in $S_{\boldsymbol{\delta}}$, to prove the equality of $d(\boldsymbol{\delta}, \mathbf{N})$ and $d\left(\boldsymbol{\delta}^{\prime}, \mathbf{N}\right)$ it suffices to show that

$$
S\left\langle k_{1}, \ldots, k_{r}\right\rangle=S\left\langle k_{r}, \ldots, k_{1}\right\rangle .
$$

We shall establish the latter equality by induction on $r$. To begin with, note that (A.1) is trivially obvious for $r=1$. Suppose now that the latter equation holds for determinants $S\langle\cdot\rangle$ of order up to $r-1$. Expanding $S\left\langle k_{1}, \ldots, k_{r}\right\rangle$ by the first column we obtain:

$$
S\left\langle k_{1}, \ldots, k_{r}\right\rangle=E_{k_{r}} S\left\langle k_{1}, \ldots, k_{r-1}\right\rangle-S\left\langle k_{1}, \ldots, k_{r-2}, k_{r-1}+k_{r}\right\rangle .
$$

Similarly, expanding $S\left\langle k_{r}, \ldots, k_{1}\right\rangle$ by the last row we have

$$
S\left\langle k_{r}, \ldots, k_{1}\right\rangle=E_{k_{r}} S\left\langle k_{r-1}, \ldots, k_{1}\right\rangle-S\left\langle k_{r-1}+k_{r}, k_{r-2}, \ldots, k_{1}\right\rangle .
$$

Equation (A.1) follows immediately from Eq. (A.2) using the induction hypothesis and Eq. (A.3). Note, finally, that the latter proof can be easily adapted to the $\mathrm{su}(n \mid m)$ case with arbitrary $n$.

\section{References}

[1] Schlottmann P, Integrable narrow-band model with possible relevance to heavy-fermion systems, 1987 Phys. Rev. B 365177

[2] Zhang F C and Rice T M, Effective Hamiltonian for the superconducting Cu oxides, 1988 Phys. Rev. B 373759

[3] Essler F H L, Korepin V E and Schoutens K, New exactly solvable model of strongly correlated electrons motivated by high-T $T_{c}$ superconductivity, 1992 Phys. Rev. Lett. 682960 
[4] Sutherland B, Model for a multicomponent quantum system, 1975 Phys. Rev. B 123795

[5] Bares P and Blatter G, Supersymmetric t-J model in one dimension: separation of spin and charge, 1990 Phys. Rev. Lett. 642567

[6] Kawakami N and Yang S K, Correlation functions in the one-dimensioanal t-J model, 1990 Phys. Rev. Lett. 652309

[7] Sarkar S, The supersymmetric t-J model in one dimension, 1991 J. Phys. A: Math. Gen. 241137

[8] Essler F H L and Korepin V E, Higher conservation laws and algebraic Bethe Ansätze for the supersymmetric t-J model, 1992 Phys. Rev. B 469147

[9] Kuramoto Y and Yokoyama H, Exactly soluble supersymmetric t-J-type model with long-range exchange and transfer, 1991 Phys. Rev. Lett. 671338

[10] Kuramoto Y, Supersymmetric long-range $t$-J model as a new canonical model for strongly correlated fermions, 1993 Physica B 186-188 831

[11] Haldane F D M, Exact Jastrow-Gutzwiller resonating-valence-bond ground state of the spin-1/2 antiferromagnetic Heisenberg chain with $1 / r^{2}$ exchange, 1988 Phys. Rev. Lett. 60635

[12] Shastry B S, Exact solution of an $S=1 / 2$ Heisenberg antiferromagnetic chain with long-ranged interactions, 1988 Phys. Rev. Lett. 60639

[13] Kawakami N and Yang S K, Finite-size scaling in one-dimensional quantum liquid with long-range interaction, 1991 Phys. Rev. Lett. 672493

[14] Sutherland B and Shastry B S, Solution of some integrable one-dimensional quantum systems, 1993 Phys. Rev. Lett. 715

[15] Kawakami N, Asymptotic Bethe-ansatz solution of multicomponent quantum systems with $1 / r^{2}$ long-range interaction, 1992 Phys. Rev. B 461005

[16] Kawakami N, Asymptotic Bethe ansatz: Application to the one-dimensional t-J model with longrange exchange and transfer, 1992 Phys. Rev. B 457525

[17] Wang D F, Liu J T and Coleman P, Spectrum and thermodynamics of the one-dimensional supersymmetric $t-J$ model with $1 / r^{2}$ exchange and hopping, 1992 Phys. Rev. B 466639

[18] Haldane F D M, Ha Z N C, Talstra J C, Bernard D and Pasquier V, Yangian symmetry of integrable quantum chains with long-range interactions and a new description of states in conformal field theory, 1992 Phys. Rev. Lett. 692021

[19] Haldane F D M, Physics of the ideal semion gas: spinons and quantum symmetries of the integrable Haldane-Shastry spin chain, in A Okiji and N Kawakami, eds., Correlation Effects in Lowdimensional Electron Systems, Springer Series in Solid-state Sciences, volume 118, pp. 3-20

[20] Basu-Mallick B, Bondyopadhaya N, Hikami K and Sen D, Boson-fermion duality in SU $(m \mid n)$ supersymmetric Haldane-Shastry spin chain, 2007 Nucl. Phys. B $\mathbf{7 8 2} 276$

[21] Saiga Y and Kuramoto Y, Dynamical properties of the one-dimensional supersymmetric $t-J$ model: a view from elementary excitations, 1999 J. Phys. Soc. Jpn. 683631

[22] Finkel F, González-López A, León I and Rodríguez M A, Thermodynamics and criticality of supersymmetric spin chains with long-range interactions, 2018 J. Stat. Mech.-Theory E. 2018 043101(47)

[23] Basu-Mallick B, Bondyopadhaya N and Hikami K, One-dimensional vertex models associated with a class of Yangian invariant Haldane-Shastry like spin chains, 2010 Symmetry Integr. Geom. 6 091(13)

[24] Inozemtsev V I, On the connection between the one-dimensional $S=1 / 2$ Heisenberg chain and Haldane-Shastry model, 1990 J. Stat. Phys. 591143

[25] Kuramoto Y and Kato Y, Spin-charge separation at finite temperature in the supersymmetric t-J model with long-range interactions, 1995 J. Phys. Soc. Jpn. 644518

[26] Basu-Mallick B and Bondyopadhaya N, Exact partition functions of $\mathrm{SU}(m \mid n)$ supersymmetric Haldane-Shastry spin chain, 2006 Nucl. Phys. B 757280

[27] Hikami K and Basu-Mallick B, Supersymmetric Polychronakos spin chain: motif, distribution function, and character, 2000 Nucl. Phys. B 566511

[28] Göhmann F and Wadati M, A note on inverse-square exchange models, 1995 J. Phys. Soc. Jpn. 


\section{5}

[29] Carrasco J A, Finkel F, González-López A, Rodríguez M A and Tempesta P, Critical behavior of $\mathrm{su}(1 \mid 1)$ supersymmetric spin chains with long-range interactions, 2016 Phys. Rev. E 93 $062103(12)$

[30] Carrasco J A, Finkel F, González-López A and Rodríguez M A, Supersymmetric spin chains with nonmonotonic dispersion relation: Criticality and entanglement entropy, 2017 Phys. Rev. E 95 $012129(15)$

[31] Calogero F and Perelomov A M, Properties of certain matrices related to the equilibrium configuration of the one-dimensional many-body problems with the pair potentials $v_{1}(x)=$ $-\log |\sin x|$ and $v_{2}(x)=1 / \sin ^{2} x, 1978$ Commun. Math. Phys. 59109

[32] Finkel F and González-López A, Global properties of the spectrum of the Haldane-Shastry spin chain, 2005 Phys. Rev. B 72 174411(6)

[33] Ha Z N C and Haldane F D M, Elementary excitations of one-dimensional $t$-J model with inverse square exchange, 1994 Phys. Rev. Lett. 732887

[34] Uimin G V, One-dimensional problem for $s=1$ with modified antiferromagnetic Hamiltonian, 1970 JETP Lett. 12225

[35] Lai C K, Lattice gas with nearest-neighbor interaction in one dimension with arbitrary statistics, 1974 J. Math. Phys. 151675

[36] Inozemtsev V I, The eigenvectors of the Heisenberg Hamiltonian with elliptic form of the exchange spin interaction, 2005 J. Nonlin. Math. Phys. 12395

[37] Finkel F and González-López A, Exact solution and thermodynamics of a spin chain with longrange elliptic interactions, 2014 J. Stat. Mech.-Theory E. 2014 P12014(28)

[38] Finkel F and González-López A, A new perspective on the integrability of Inozemtsev's elliptic spin chain, 2014 Ann. Phys.-New York 351797

[39] Polychronakos A P, Lattice integrable systems of Haldane-Shastry type, 1993 Phys. Rev. Lett. 70 2329

[40] Polychronakos A P, Exact spectrum of SU(n) spin chain with inverse-square exchange, 1994 Nucl. Phys. B 419553

[41] Finkel F and González-López A, Yangian-invariant spin models and Fibonacci numbers, 2015 Ann. Phys.-New York $\mathbf{3 6 1} 520$

[42] Kirillov A N, Kuniba A and Nakanishi T, Skew Young diagram method in spectral decomposition of integrable lattice models, 1997 Commun. Math. Phys. 185441

[43] Enciso A, Finkel F and González-López A, Thermodynamics of spin chains of Haldane-Shastry type and one-dimensional vertex models, 2012 Ann. Phys.-New York 3272627 